\title{
Chern-Simons theory on Seifert manifold and matrix model
}

\author{
Arghya Chattopadhyay $\odot,{ }^{*}$ Suvankar Dutta, ${ }^{\dagger}$ and Neetu \\ Department of Physics, Indian Institute of Science Education and Research Bhopal, Bhopal 462 066, India
}

(Received 14 May 2019; published 6 December 2019)

\begin{abstract}
Chern-Simons (CS) theories with rank $N$ and level $k$ on Seifert manifold are discussed. The partition functions of such theories can be written as a function of modular transformation matrices summed over different integrable representations of affine Lie algebra $u(N)_{k}$ associated with the boundary WessZumino-Witten model. Using properties of modular transform matrices we express the partition functions of these theories as a unitary matrix model. We show that the eigenvalues of unitary matrices are discrete and proportional to hook lengths of the corresponding integrable Young diagram. As a result, in the large $N$ limit, the eigenvalue density develops an upper cap. We consider CS theory on $S^{2} \times S^{1}$ coupled with fundamental matters and express the partition functions in terms of modular transformation matrices. Solving this model at large $N$ we find the dominant integrable representations and show how large $N$ representations are related to each other by transposition of Young diagrams as a result of level rank duality. Next we consider $U(N)$ CS theory on $S^{3}$ and observed that in Seifert framing the dominant representation is no longer an integrable representation after a critical value of 't Hooft coupling. We also show that CS on $S^{3}$ admits multiple (two-gap phase) large $N$ phases with the same free energy.
\end{abstract}

DOI: 10.1103/PhysRevD.100.126009

\section{INTRODUCTION}

Study of topological objects in physics is an extremely interesting subject. One of the earliest examples of topological objects is the Dirac monopole [1]. Today we know a large number of such examples in physics starting from quantum mechanics to string theory. An example of such objects in mathematics is the knot (closed path). Mathematically, a knot is just a smooth closed, nonselfintersecting curve in three dimensions. Knot theory turns out to be especially useful to study the physics of twodimensional many body systems [2,3]. A field theoretic realization of knots and links (collection of nonintersecting knots) was discovered by Witten in his groundbreaking work in 1989 [4]. In that paper he showed that physical observables (Wilson loops) in Chern-Simons (CS) gauge theory in three dimensions are related to knot polynomials in the same dimensions and thus opened up a plethora of new possibilities for both mathematicians and physicists.

To discuss the connection in a little detail, we start with pure Chern-Simons theory with gauge group $G$ and

\footnotetext{
*arghya@iiserb.ac.in suvankar@iiserb.ac.in

neetuj@iiserb.ac.in
}

Published by the American Physical Society under the terms of the Creative Commons Attribution 4.0 International license. Further distribution of this work must maintain attribution to the author(s) and the published article's title, journal citation, and DOI. Funded by SCOAP ${ }^{3}$. level $k$ on a compact three manifold $\mathbb{M}$. The action is given by

$S_{\mathrm{CS}}[\mathbb{M}, G, k]=\frac{k}{4 \pi} \int_{\mathbb{M}} \operatorname{Tr}\left(A \wedge d A+\frac{2}{3} A \wedge A \wedge A\right)$.

The action does not require any metric on $\mathbb{M}$. This defines the most simple version of topological field theories, namely, the Schwarz-type topological field theory. The partition function for Chern-Simons theory on $\mathbb{M}$,

$$
\mathcal{Z}_{\mathrm{CS}}[\mathrm{M}, G, k]=\int[D A] e^{i S_{\mathrm{CS}}}
$$

itself defines a topological invariant of the manifold $\mathbb{M}$. To explain the relation between observables in Chern-Simons theory and knot polynomials we consider Wilson loop operators in representation $R$ of $G$ along an oriented knot $\mathcal{K}$ in $\mathbb{M}$, defined as

$$
\mathcal{W}_{R}^{\mathcal{K}}(A)=\operatorname{Tr}_{R} U_{\mathcal{K}} \quad \text { with } \quad U_{\mathcal{K}}=P \exp \oint_{\mathcal{K}} A
$$

where $U_{\mathcal{K}}$ is called holonomy around the knot $\mathcal{K}$. Since Wilson loop operators are gauge invariant by definition and in this particular case they are metric independent as well, it is quite obvious that the correlation functions of the Wilson loop operators 


$$
\left\langle\mathcal{W}_{R_{1}, \cdots, R_{n}}^{\mathcal{K}_{1}, \ldots, \mathcal{K}_{n}}\right\rangle=\int[D A] e^{i S_{\mathrm{CS}}} \prod_{i=1}^{n} \mathcal{W}_{R_{i}}^{\mathcal{K}_{i}}(A)
$$

generate topological invariants of the theory. Witten [4] proved that these topologically invariant correlation functions are precisely the knot invariants. ${ }^{1}$

There is a subtle caveat in this seemingly simple story. The metric independence of the classical Lagrangian does not trivially generalize to the quantum version of the theory. Witten showed that the quantum version of Chern-Simons theory preserves topological invariance but at the expense of a choice of "framing." Correlation functions of Wilson loop operators along different knots depend on the linking number between the knots involved in the computation [4,5]. The linking number between two knots is a topologically invariant quantity; therefore, in general the correlation functions are also topologically invariant. But the subtlety arises when different Wilson loop operators are taken along the same knot. Then the notion of framing becomes important because in general the self-linking number $^{2}$ of a knot $\mathcal{K}$ is not a topologically invariant quantity. Therefore, to preserve topological invariance of the correlation function one needs to modify the definition of self-linking number by a choice of framing $[4,5]$. To generate a consistent notion of self-linking number, one defines another knot $\mathcal{K}_{f}$ around $\mathcal{K}$ specified by some normal vector field $n$ and defines self-linking number as the linking number between $\mathcal{K}$ and $\mathcal{K}_{f}$. This manner of regularization reinstates topological invariance of correlation functions but at the expense of its dependence on some integer $p$ defining the linking number between $\mathcal{K}$ and $\mathcal{K}_{f}$. To visualize the situation, we can imagine the set of normal vectors defined by the vector field $n$, as a tangled ribbon defining an orientable surface, the sides of which are bounded by $\mathcal{K}$ and $\mathcal{K}_{f}$. In principle, there are many ways to construct such ribbons so that one side always coincides with $\mathcal{K}$, and each of these choices renders different framings of the knot. The canonical framing is defined as some choice of $n$ such that the self-linking number $p$ is 0 . The framing that is very crucial for this paper is called the Seifert framing, where the knot is pushed along the Seifert surface ${ }^{3}$ to generate $\mathcal{K}_{f}$ for regularization. Now it is obvious that a change of framing is nothing but changing the choice of the vector field $n$, which just renders a change in the value of the integer $p$ defined above. Following [4] it can be shown that, under a change of framing of $\mathcal{K}_{i}$ by $p_{i}$ units, the correlation function of the Wilson loops changes as

\footnotetext{
${ }^{1}$ For $G \equiv U(N)$ or $G \equiv S U(2)$ or $G \equiv S O(N)$ these correlation functions are in turn related to the HOMFLY polynomial or the Jones polynomial or Kauffman polynomials, respectively.

${ }^{2}$ Also known as the cotorsion or the writhe.

${ }^{3}$ A compact, connected, oriented surface embedded in the three manifold having the knot $\mathcal{K}$ as its boundary such that the orientation of the knot is consistent with its own.
}

$$
\left\langle\mathcal{W}_{R_{1}, \cdots R_{n}}^{\mathcal{K}_{1}, \ldots, \mathcal{K}_{n}}\right\rangle \rightarrow \exp \left(2 \pi i \sum_{i=1}^{n} p_{i} h_{R_{i}}\right)\left\langle\mathcal{W}_{R_{1}, \cdots R_{n}}^{\mathcal{K}_{1}, \ldots, \mathcal{K}_{n}}\right\rangle
$$

where $h_{R_{i}}=\frac{C_{R_{i}}}{2(k+N)}$ with $c_{R_{i}}$ being the quadratic Casimir in the representation $R_{i}$ of $G(N)$.

Not only the correlators but also the partition function depends on choice of framing. Atiyah [6] showed that for every three manifold $\mathbb{M}$ different framing choices can be labeled by an integer $s \in \mathbb{Z}$ ( $s$ is a self-linking number) such that the canonical framing is given by $s=0$. As a result, if two framings differ by an integer $s$, the corresponding partition functions are related by [5]

$\mathcal{Z}_{\mathrm{CS}}[\mathrm{M}, G, k]=\exp \left(\frac{\pi i s c}{12}\right) \mathcal{Z}_{\mathrm{CS}}[\mathbb{M}, G, k] ; \quad c=\frac{k d}{k+y}$,

where $d$ and $y$ are the dimension and dual Coxeter number of the group $G$, and $c$ is the central charge of the WessZumino-Witten (WZW) model $^{4}$ with the affine gauge group $G_{k}$. One should realize how the WZW model naturally arises in the context of quantizing Chern-Simons theory on three manifolds. The CS/WZW correspondence is in some sense a predecessor of AdS/CFT. The status of AdS/CFT is still at the level of a conjecture, whereas one can show that Chern-Simons theory quantized on a closed three manifold can be described exactly by a two-dimensional WZW model. ${ }^{5}$ Since the CS/WZW is an exact correspondence, one can write observables in Chern-Simons theory in terms of objects in the dual WZW model. ${ }^{6}$ These objects are modular transformation matrices [7] of the affine lie algebra. This precise relation is our starting point in this paper. We illustrate this relation in detail in the subsequent section.

\footnotetext{
${ }^{4}$ See [7] for a pedagogical review of WZW and [4] for a detailed relation between Chern-Simons theory and the WZW model.

${ }^{5}$ For a comparison between the CS/WZW with $\mathrm{AdS}_{3} / \mathrm{CFT}_{2}$ one can look at the beautiful paper by Gukov et al. [8].

${ }^{6}$ The central statement that connects these two completely different theories in two different dimensions is that the Hilbert space that one gets while quantizing the $(2+1)$-dimensional CS theory turns out to be the space of conformal blocks for the $(1+1)$-dimensional WZW theory. For example, if one starts quantizing a pure level $k \mathrm{CS}$ theory with gauge group $G$ on $\Sigma_{g} \times S^{1}$, then the physical Hilbert space of CS theory $H_{\Sigma}$ turns out to be finite dimensional and can be described as the space of conformal blocks of a WZW theory on $\Sigma_{g}$ with the affine gauge group $G_{k}$. As spelled out by [4] one can generalize this result to a generic Seifert manifold $\mathcal{M}_{(g, p)}$ [a circle bundle over $\Sigma_{g}$ with first Chern class $p$. Ex. $S^{3} / \mathbb{Z}_{p}$ for $\left.(g, p)=(0, p)\right]$ by doing surgeries over $\Sigma_{g} \times S^{1}$. In fact different choices of doing surgery to go from one manifold to the other results in generating the same partition function but in a different choice of framings. A lot of work has been done on Chern-Simons theories on Seifert manifolds, the most recent of which is [9], and one can look at the references therein for other related works.
} 
In this paper we study large $N$ properties of ChernSimons theory with level $k$ and gauge group $G=S U(N)$ or $U(N)^{7}$ on Seifert manifold $\mathcal{M}_{(g, p)}$. The partition function for the same in Seifert framing can be written as a function of modular transform matrices ${ }^{8}$ of affine Lie group summed over integrable representations. An integrable representation of $u(N)_{k}$ (or $s u(N)_{k}$ ) has maximum $k$ columns and $N$ rows in the Young diagram. The restriction on representations follows from the fact that there is a precise relation between Hilbert space $\mathcal{H}_{\Sigma}$ of Chern-Simons theory with level $k$ and gauge group $S U(N)$ on $\Sigma \times S^{1}$ and the WZW model on $\Sigma$ with affine group $S U(N)_{k}$. The Hilbert space $\mathcal{H}_{\Sigma}$ is finite dimensional and spanned by the finite number of conformal primaries in the WZW model. In the WZW model the conformal primaries are finite in number and in one-to-one correspondence with integrable representations of affine Lie algebra $s u(N)_{k}$. The first goal of this paper is to show that using the form of modular transform matrices $\mathcal{S}$ and $\mathcal{T}$ for given representations, the partition function can be written as a unitary matrix model. These matrix models are similar to those studied by $[10,11]^{9}$ but with a difference. We observe that the eigenvalues of unitary matrices are proportional to hook numbers associated with integrable representations. As a result the eigenvalues turn out to be discrete.

To check the consistency of our observation we consider the CS theory on $S^{2} \times S^{1}$ coupled with Gross-WittenWadia (GWW) potential $[12,13] .{ }^{10}$ The resulting unitary matrix model turns out to be exactly the same as the one derived earlier by [16] in the context of CS theory coupled with matter. The authors of [16] also showed that the eigenvalues of unitary matrices are discrete and the discreteness in eigenvalues comes because of $U(1)$ fluxes through $S^{2}$. In our way of writing the partition function it seems that discreteness in eigenvalues emerges naturally.

\footnotetext{
${ }^{7}$ In this paper we work in large $N$ limit. Our results are not sensitive to this choice.

${ }^{8}$ The characters of the integrable representations of an affine Lie algebra $s u(N)_{k}$ transform into one another under modular transformations. The two generators of this modular group are conventionally denoted by $\mathcal{S}$ and $\mathcal{T}$. See Sec. II for details.

${ }^{9}$ The partition function for CS theories on different manifolds boils down to a novel class of matrix models [10], with a unitary matrix model (UMM)-like measure and a nonperiodic potential.

${ }^{10}$ This partition function of Chern-Simons theory on $S^{2} \times S^{1}$ can also be written in terms of a sum over representations of $S U(N)[14,15]$. The $S U(N)$ representations are characterized by Young diagrams with maximum $N$ rows (with no constraint on the maximum number of columns). In our previous work [15] we observed that the discreteness in eigenvalue distribution imposes a constraint on the dominant representations of $S U(N)$ : The maximum number of columns must be less than $k$, which is nothing but the integrability condition. This observation motivated us to look at the relation between CS theories on different manifolds directly starting from its relation with the current algebra of the corresponding WZW theory. We postpone further discussion on this to the conclusion, Sec. V.
}

In a series of papers [17-21] Naculich et al. showed how the level-rank duality of the WZW model flows to the level-rank duality of Chern-Simons theories. Under level rank duality a Wilson loop in one theory with some representation characterized by the Young diagram $Y$ maps to a Wilson loop with a representation $\tilde{Y}$ in the level-rank dual theory, where $Y$ and $\tilde{Y}$ are related by transposition. ${ }^{11}$ Since the GWW model is self-dual, writing down the partition function as a sum over integrable representations, we explicitly check that different dominant representations at large $N$ are related to each other by transposition. In this paper we consider not only GWW potential, Chern-Simons theory of $S^{2} \times S^{1}$ coupled with fundamental matter. The partition function for such theory, in large $N$ limit, can be written as the expectation value of an effective function of Wilson loops in pure Chern-Simons theory $[16,22]$. We express the partition function for these theories in terms of modular transformation matrices summed over integrable representations. This might help us understand the relation between CS theory coupled with different fundamental matters using the transformation properties of modular transformation matrices under level-rank duality.

In this paper we also study pure level $k$, rank $N$ ChernSimons theory on $S^{3}$. The partition function for this theory in canonical framing is trivial and given by the 00 component $^{12}$ of modular transformation matrix $\mathcal{S}$. The free energy at large $N$ matches with topological string theory on the resolved conifold [23] and exhibits no phase transition. The same partition function in Seifert framing can be written as function of modular transform matrices summed over integrable representations. Using the properties of modular transform matrices one can show the equivalence between two framings up to a phase factor. However, the latter admits a matrix model representation of the theory. In this paper we show that the partition function can be written as a unitary matrix model where eigenvalues are discrete. The discreteness implies an upper cap in eigenvalue distribution in large $N$ limit. In our analysis we see that at large $N$ the partition function is dominated by a one-gap eigenvalue distribution that corresponds to an integrable representation and large $N$ free energy matches with the same in canonical framing. However, the large $N$ phase ceases to exist after a critical value of 't Hooft coupling $\lambda$. This implies that for 't Hooft coupling greater than the critical value the most dominant representation is not an integrable representation anymore. We do not have any satisfactory explanation for the existence of such critical value of 't Hooft coupling in the theory.

While studying large $N$ phases of pure Chern-Simons theory on $S^{3}$ we encounter another interesting phase of the model. We observe that for a range of 't Hooft coupling there exists a new phase of the system with similar free

\footnotetext{
${ }^{11}$ Though this mapping is not in general one to one [21].

${ }^{12} 0$ representation means a Young diagram with no box.
} 
energy. The new phase corresponds to a two-gap distribution. Interestingly, the new phase (two-gap phase) also ceases to exist after the same critical value of 't Hooft coupling. However, we failed to find the new phase for lower values of 't Hooft coupling. This could be because of our limitation in numerical analysis. The bottom line is, for a finite range of 't Hooft coupling we observe that at large $N$ Chern-Simons theory on $S^{3}$ admits two topologically distinct eigenvalue distributions with same free energy. It would be interesting to understand the meaning of this new phase in the topological string theory side [23].

The plan of this paper is as follows. In Sec. II we discuss how the partition function for Chern-Simons theory on Seifert manifold can be written as a function of modular transform matrices summed over integrable representations and their dependence on framings. We also show that using the expressions for modular transform matrices one can write this partition function as a unitary matrix model. In Sec. III we consider Chern-Simons theory on $S^{2} \times S^{1}$ coupled with different fundamental matter. We write down the partition function in terms of modular transform matrices for any generic fundamental matter coupling. As a toy model we consider Chern-Simons theory coupled with GWW potential and find different dominant integrable representations for different phases of the theory at large $N$. From the dominant representations it is manifest that the theory is self-dual under level-rank duality. Section IV contains discussion Chern-Simons theory on $S^{3}$. We show that at large $N$ there is a discrepancy in writing the partition function in Seifert framing. Namely, restriction on integrable representations seems to break down after a critical value of 't Hooft coupling. We summarize our main results in conclusion Sec. V and discuss how the dominant representations found in the current paper are different than what we considered in our previous works $[14,15]$.

\section{CHERN-SIMONS PARTITION FUNCTION ON THE SEIFERT MANIFOLD}

In this section we discuss how one can write the partition function (or correlation of Wilson loops) of a generic Chern-Simons theory on a three-dimensional compact manifold as a unitary matrix model.

We consider Chern-Simons theory of level $k$ (bare level) and gauge group $G(N)$ on a Seifert manifold. A Seifert manifold $\mathcal{M}_{(g, p)}$ is a circle bundle over genus $g$ Riemann surface $\Sigma_{g}$ with first Chern class $p$. Physical observables (Wilson loops) of Chern-Simons theory also on such a manifold can be written in terms of observables in twodimensional WZW theory because of close connection between the two [4]. A Seifert manifold for generic $p$ can be obtained from $\mathcal{M}_{(g, 0)}$ (which is a product of genus $g$ Riemann surface and a circle $\Sigma_{g} \times S^{1}$ ) by surgery. Different choices of surgery give different framings of $\mathcal{M}_{(g, p)}$. In a particular framing called Seifert framing, the expectation value of $n$ Wilson loops in different representations $\mathcal{R}_{1}, \mathcal{R}_{2}, \ldots, \mathcal{R}_{n}$ of $G(N)$ can be written as $[21,24]$

$\mathcal{W}_{\mathcal{R}_{1}, \ldots, \mathcal{R}_{n}}\left[\mathcal{M}_{(g, p)}, G, k\right]=\sum_{\mathcal{R}}\left(\mathcal{T}_{\mathcal{R} \mathcal{R}}\right)^{-p} \mathcal{S}_{0 \mathcal{R}}^{2-n-2 g} \prod_{i=1}^{n} \mathcal{S}_{\mathcal{R} \mathcal{R}_{i}}$,

where $\mathcal{T}_{\mathcal{R R}^{\prime}}$ and $\mathcal{S}_{\mathcal{R R}^{\prime}}$ are modular transform matrices that mix the affine characters associated with highest weight representations of affine Lie algebra $g(N)_{k}$ under translation and inversion of modular parameter $\tau$, respectively,

$$
\begin{aligned}
& \chi_{\mathcal{R}}(\tau+1)=\sum_{\mathcal{R}^{\prime}} \mathcal{T}_{\mathcal{R R}^{\prime} \chi_{\mathcal{R}^{\prime}}(\tau) \quad \text { and }} \\
& \chi_{\mathcal{R}}(-1 / \tau)=\sum_{\mathcal{R}^{\prime}} \mathcal{S}_{\mathcal{R}^{\prime}} \chi_{\mathcal{R}^{\prime}}(\tau) .
\end{aligned}
$$

All the sums in Eqs. (2.1) and (2.2) are over integrable representations of $g(N)_{k}$ and $\mathcal{R}=0$ corresponds to identity representation. As mentioned earlier, we express all the representations in terms of the Young diagram and hence an integrable representation means the Young diagram with maximum $k$ number of boxes in the first row (i.e., maximum $k$ columns).

For $G=U(N)$ the modular transform matrix $\mathcal{S}_{\mathcal{R} \mathcal{R}^{\prime}}$ of the $u(N)_{k}$ can be written in terms of modular transformation matrix of $s u(N)$ and is given by ${ }^{13}$ [25]

$\mathcal{S}_{\mathcal{R R}^{\prime}}=(-i)^{\frac{N(N-1)}{2}}(k+N)^{-N / 2} e^{-\frac{2 \pi i Q Q^{\prime}}{N(N+k)}} \operatorname{det} M\left(R, R^{\prime}\right)$.

We use the notation $\mathcal{R}$ and $R$ for $u(N)$ and $\operatorname{su}(N)$ representations, respectively. ${ }^{14} M\left(R, R^{\prime}\right)$ is an $N \times N$ matrix with elements

$$
M_{i j}\left(R, R^{\prime}\right)=\exp \left[\frac{2 \pi i}{k+N} \phi_{i}(R) \phi_{j}\left(R^{\prime}\right)\right],
$$

where

$\phi_{i}(R)=h_{i}-\frac{s^{R}}{N}, \quad s^{R}=\sum_{i=1}^{N} h_{i}$ and $h_{i}=n_{i}+N-i$.

$n_{i}$ 's are number of boxes in $i$ th row of a given representation $R$ and $n_{1} \leq k$ with $R$ being an integrable representation. $Q$ is the eigenvalue of the $u(1)$ generator and is given by $Q=r(R) \bmod N ; r(R)$ is the number of boxes in $R$.

\footnotetext{
${ }^{13}$ Affine Lie algebra of the $U(N)$ WZW model is the quotient of $s u(N)_{k} \times u(1)_{N(k+N)}$ by $\mathbb{Z}_{N}$. Hence $u(N)$ representation can be written in terms of $s u(N)$ representations and eigenvalues of the $u(1)$ generator.

${ }^{14}$ One can follow [26] for a generic discussion and derivation of modular transformation matrices of affine Lie algebra.
} 
After a little algebra, the $(0, \mathcal{R})$ component of modular transform matrix $\mathcal{S}$ can be written in terms of hook numbers $h_{i}$ 's,

$$
\mathcal{S}_{0 \mathcal{R}}=(k+N)^{-N / 2} 2^{N(N-1) / 2} e^{-\frac{2 \pi i Q(\mathcal{R}) Q(0)}{N(N+k)}} \prod_{i<j} \sin \left(\frac{\pi\left(h_{i}-h_{j}\right)}{k+N}\right) .
$$

$\mathcal{R}=0$ means identity representation, i.e., $n_{i}=0, \forall i \in$ $[1, N]$. As we see, this expression plays an important role in our analysis.

The other modular transform matrix $\mathcal{T}_{\mathcal{R R}^{\prime}}$ is given by

$$
\begin{aligned}
\mathcal{T}_{\mathcal{R R}^{\prime}} & =\exp \left(2 \pi i\left(h_{R}-\frac{c}{24}\right)\right) \delta_{\mathcal{R R}^{\prime}}, \\
h_{R} & =\frac{1}{2} \frac{C_{2}(\mathcal{R})}{k+N}, \quad c=\frac{N(N k+1)}{k+N}
\end{aligned}
$$

and $\mathcal{C}_{2}(\mathcal{R})$ is quadratic Casimir of $u(N)_{k}$,

$$
C_{2}(\mathcal{R})=N \sum_{i=1}^{N}\left(l_{i}+s\right)+\sum_{i=1}^{N}\left(l_{i}+s\right)\left(\left(l_{i}+s\right)-2 i+1\right) .
$$

Here $s$ is any integer. Young diagram $\mathcal{R}$ of $u(N)$ is obtained by prepending $s$ columns of $N$ boxes to the Young tableaux $R$ for the corresponding $S U(N)$. Thus the number of boxes in $\mathcal{R}$ is given by $l_{i}+s$ and hence can be negative as well. However, we work in terms of the number of boxes of the corresponding $s u(N)$ representations $R$.

The partition function for Chern-Simons theory on $\mathcal{M}_{(g, p)}$ can be written from Eq. (2.1) setting $n=0$,

$$
\mathcal{Z}_{\mathrm{CS}}\left[\mathcal{M}_{(g, p)}, U(N), k\right]=\sum_{\mathcal{R}}\left(\mathcal{T}_{\mathcal{R} \mathcal{R}}\right)^{-p} \mathcal{S}_{0 \mathcal{R}}^{2-2 g}
$$

$\mathcal{R}$ runs over integrable representations.

We work with this partition function in this paper.

\section{A. unitary matrix model and large $N$ limit}

Starting with the above partition function (2.9), one can express the same in terms of hook number variables $\left\{h_{i}\right\}$,

$$
\begin{aligned}
\mathcal{Z}_{\mathrm{CS}}\left[\mathcal{M}_{(g, p)}, G, k\right]= & \left(\frac{2^{(N-1)}}{k+N}\right)^{N(1-g)} \\
& \times \sum_{\vec{h}} \prod_{i<j} \sin ^{2-2 g}\left(\frac{\pi\left(h_{i}-h_{j}\right)}{k+N}\right) \\
& \times e^{-\frac{i p \pi}{k+N} \sum_{i}\left(h_{i}-\Delta\right)^{2}} e^{\frac{\pi i p N^{2}}{12}-\frac{2(2-2 g) i \pi Q(R) Q(0)}{N(k+N)}}, \\
\text { where } \Delta= & \frac{1}{2}(N-1-2 s) \text { and } \\
\sum_{\vec{h}}= & \sum_{\substack{\left\{h_{i}\right\}=0 \\
h_{1}>h_{2}>\cdots \rightarrow h_{N}}}^{k+N} \text { is a restricted sum. }
\end{aligned}
$$

Here, $Q(R)=r(R)+s N$ and $Q(0)=s^{\prime} N, r(R)$ being the number of boxes in $R$ representation. Since summation in Eq. (2.10) is over the integrable representations, the hook number $h_{i}$ ranges between 0 and $k+N$. To write the partition function in terms of a unitary matrix model we impose the periodic boundary condition on $h_{i}: h_{i} \sim h_{i}+k+N$. We define angular variables $\left\{\theta_{i}\right\}$ as

$\theta_{i}=\left(\frac{2 \pi}{k+N}\right) h_{i}$, where $\theta_{N} \geq 0$ and $\theta_{1} \leq 2 \pi$.

Periodicity in $h_{i}$ implies $\theta_{i} \sim \theta_{i}+2 \pi$. Here, note that angular variables $\left\{\theta_{i}\right\}$ 's are in monotonically decreasing order. In the large $N$ limit, Eq. (2.10) can be written in terms of redefined variables $\theta_{i}$ 's as

$$
\begin{aligned}
& \mathcal{Z}_{\mathrm{CS}}\left[\mathcal{M}_{(g, p)}, G, k\right] \\
& =2^{N(N-1)} \int \prod_{i} \frac{d \theta_{i}}{2 \pi} \prod_{i<j} \sin ^{2-2 g}\left(\frac{\theta_{i}-\theta_{j}}{2}\right) e^{f\left(\left\{\theta_{i}\right\}, k, N, p\right)}
\end{aligned}
$$

with some effective potential $f\left(\theta_{i}, k, N, p\right)$ depending on angular variable $\left\{\theta_{i}\right\}$ and other parameters. Thus, we see that the partition function can be written as a unitary matrix model with an effective potential $f\left(\left\{\theta_{i}\right\}, k, N, p\right)$. The modular transform matrix $\mathcal{S}_{0 \mathcal{R}}$ provides the correct measure factor for CS theory on any compact Riemann surface of genus $g[16,24]$.

From the redefinition (2.11) we see that eigenvalues are discrete (since hook numbers can take only integer values). The discreteness of eigenvalues for Chern-Simons theory on $S^{2} \times S^{1}$ was discussed in [16] and the source of the discreteness was the $U(1)$ flux through $S^{2}$. Here we see that the discreteness is automatic for any three manifold (not just $S^{2} \times S^{1}$ ) when we write the Chern-Simons partition function as a sum over integrable representations of the $u(N)_{k}$ WZW model.

Discreteness in eigenvalues implies an upper cap in eigenvalue distribution function ${ }^{15}$ defined as

\footnotetext{
${ }^{15}$ We use a negative sign in the definition of eigenvalue density because we arrange the eigenvalues in Eq. (2.10) in monotonically decreasing order.
} 
$\rho(\theta)=-\lim _{\Delta x \rightarrow 0} \frac{\Delta x}{\Delta \theta}$, where $x=i / N$ and $\theta(x)=\theta_{i}$.

In the large $N$ limit

$$
\rho(\theta) \leq \frac{1}{2 \pi \lambda}, \quad \text { where } \lambda=\frac{N}{k+N} .
$$

In large $N$ limit, we can also define a Young diagram distribution function $u(h)$,

$u(h)=-\lim _{\Delta x \rightarrow 0} \frac{\Delta x}{\Delta h}, \quad$ where $x=i / N$ and $h(x)=h_{i} / N$.

Thus the Young distribution and eigenvalue distributions are related by

$$
u(h)=2 \pi \lambda \rho(2 \pi \lambda h) .
$$

From the relation $n_{i}=h_{i}-N+i$ we can independently check that the above $u(h)$ satisfies

$$
u(h) \leq 1
$$

since $n_{i}$ 's are monotonically decreasing numbers. Therefore, this bound on $u(h)$ is consistent with the identification (2.11) and the bound on eigenvalue density (2.14). Thus we see that in the large $N$ limit, finding the most dominant Young diagram is equivalent to solving the saddle point equation in the unitary matrix model. We should note that redefined variables $\theta_{i}$ 's are periodic after we impose periodic boundary condition on hook numbers $h_{i}$.

\section{CHERN-SIMONS THEORY ON $S^{2} \times S^{1}$ COUPLED WITH THE FUNDAMENTAL MATTER FIELD}

In this section we write down the partition function of $U(N)$ Chern-Simons theory of level $k$ on $S^{2} \times S^{1}$ coupled with different fundamental fields in terms of modular transform matrices of affine Lie algebra of boundary WZW model and study the properties of large $N$ representations that dominate the partition function.

It was shown in [22] that by integrating out the massive modes, the thermal partition function of large $N$ ChernSimons theory on $S^{2} \times S^{1}$ can be written in terms of the vacuum expectation value of the effective action of holonomy $U$ along the thermal circle $S^{1}$,

$$
\mathcal{Z}_{\mathrm{CS}}^{\text {matter }}\left[S^{2} \times S^{1}, U(N), k\right]=\left\langle e^{-T^{2} V_{2} v(U)}\right\rangle_{N, k},
$$

where $T$ is the temperature (inverse of the size of the thermal circle), $V_{2}$ is the volume of $S^{2}$, and the effective potential $v(U)$ depends on the matter coupling.
The effective potential $v(U)$ can be computed case by case. In most of the cases, one can see that $v(U)$ can be written as a generic single plaquette model given by

$\mathcal{Z}(\beta)=\int \mathcal{D} U \exp \left[N \sum_{n=1}^{\infty} \frac{\beta_{n}(\beta)}{n}\left(\operatorname{Tr} U^{n}+\operatorname{Tr} U^{\dagger n}\right)\right]$,

where $\beta_{n}(\beta)$ 's are the parameters of the model. We now explicitly spell out how to write CS theory coupled with matter in the fundamental representation as an effective single plaquette model for four cases, namely, CS coupled to regular bosons, regular fermions, critical bosons, and critical fermions.

(i) Chern-Simons theory coupled to regular bosons

Consider Chern-Simons theory coupled to massless fundamental bosons or regular bosons with $\phi^{6}$ interaction [27]. The action is given by

$$
S=S_{\mathrm{CS}}+S_{R B}
$$

where $S_{R B}$ is the matter action and is given by

$$
S_{R B}=\int d^{3} x\left[\left(D_{\mu} \phi\right)^{\dagger}\left(D^{\mu} \phi\right)+\frac{\lambda_{6}}{3 ! N^{2}}\left(\phi^{\dagger} \phi\right)^{3}\right],
$$

with $\lambda_{6}$ being the marginal coupling constant. The effective potential $v[\rho]$ for this theory as a functional of eigenvalue density function $\rho(\theta)$ is given by [16]

$$
\begin{aligned}
v[\rho]= & -\frac{N}{6 \pi}\left(1+\frac{2}{\hat{\lambda}}\right) \sigma^{3} \\
& +\frac{N}{2 \pi} \int_{-\pi}^{\pi} d \theta \rho(\theta) \int_{\sigma}^{\infty} d y y\left(\ln \left(1-e^{-y+i \theta}\right)\right. \\
& \left.+\ln \left(1-e^{-y-i \theta}\right)\right),
\end{aligned}
$$

where

$$
\hat{\lambda}=\sqrt{\frac{\lambda_{6}}{8 \pi^{2}}+\lambda^{2}}
$$

and $\lambda=N /(N+k)$ is the 't Hooft coupling.

Using the following expansion (since $\left|e^{-y+i \theta}\right|<1$ for $y>0$ )

$$
\begin{array}{r}
\ln \left(1-e^{-y+i \theta}\right)+\ln \left(1-e^{-y-i \theta}\right) \\
=-\sum_{n=1}^{\infty} \frac{e^{-n y}}{n}\left(e^{i n \theta}+e^{-i n \theta}\right),
\end{array}
$$

and integrating over $y$ Eq. (3.4) can be recast as

$$
v[U]=-\frac{N}{6 \pi}\left(1+\frac{2}{\hat{\lambda}}\right) \sigma^{3}-\frac{1}{2 \pi} \sum_{n=1}^{\infty} \frac{\beta_{n}}{n}\left(\operatorname{Tr} U^{n}+\operatorname{Tr} U^{\dagger n}\right),
$$


where

$$
\beta_{n}=\frac{e^{-n \sigma}(1+n \sigma)}{n^{2}} .
$$

The value of $\sigma$ is obtained from minimization of $v[U]$ with respect to $\sigma$ in a given phase

$$
\left(1+\frac{2}{\hat{\lambda}}\right) \sigma=\frac{1}{N} \sum_{n=1}^{\infty} \frac{e^{-n \sigma}}{n}\left(\rho_{n}+\rho_{-n}\right),
$$

where $\rho_{n}=\operatorname{Tr} U^{n}$.

(ii) Chern-Simons theory coupled to regular fermions

Consider now a theory of single massless fundamental fermion minimally coupled to a $U(N)$ level $k$ Chern-Simons theory. The action is given by

$$
S=S_{\mathrm{CS}}+S_{R F},
$$

where the matter action $S_{R F}$ is given by [28]

$$
S_{R F}=\int d^{3} x \bar{\psi} \gamma^{\mu} D_{\mu} \psi
$$

Following the same steps as in the case of fundamental bosons, the effective potential $v[\rho]$ given in $[16,22]$ can be written as

$v[U]=-\frac{N}{6 \pi}\left(\frac{\tilde{c}^{3}}{\lambda}-\tilde{c}^{3}\right)-\frac{1}{2 \pi} \sum_{n=1}^{\infty} \frac{\beta_{n}}{n}\left(\operatorname{Tr} U^{n}+\operatorname{Tr} U^{\dagger n}\right)$,

where

$$
\beta_{n}=\frac{(-1)^{n+1}}{n^{2}}(1+n \tilde{c}) e^{-n \tilde{c}}
$$

The value of $\tilde{c}$ is determined by extremizing $v[\rho]$ with respect to $\tilde{c}$,

$\tilde{c}\left(\frac{1}{\lambda}-1\right)=-\frac{1}{N} \sum_{n=1}^{\infty} \frac{(-1)^{n} e^{-n \tilde{c}}}{n}\left(\rho_{n}+\rho_{-n}\right)$.

(iii) Chern-Simons theory coupled to critical bosons

At large $N$, critical bosonic theory can be defined as the Legendre transform of regular bosonic theory [Eq. (3.3) with $\lambda_{6}=0$ ] with respect to the operator $\phi^{\dagger} \phi$. The action of regular boson theory is deformed by a mass squared parameter $A$ so that the action becomes

$$
S=S_{\text {Scalar }}+S_{\mathrm{CS}}+\delta S,
$$

where

$$
\delta S=\int d^{3} x A \phi^{\dagger} \phi
$$

The effective potential [16] can be written as

$v[U]=-\frac{N}{6 \pi} \sigma^{3}-\frac{1}{2 \pi} \sum_{n=1}^{\infty} \frac{\beta_{n}}{n}\left(\operatorname{Tr} U^{n}+\operatorname{Tr} U^{\dagger n}\right)$,

where

$$
\beta_{n}=\frac{e^{-n \sigma}}{n^{2}}(1+n \sigma) .
$$

Extremizing the effective potential with respect to $\sigma$, we get

$$
\sigma=\frac{1}{N} \sum_{n=1}^{\infty} \frac{e^{-n \sigma}}{n}\left(\rho_{n}+\rho_{-n}\right) .
$$

(iv) Chern-Simons theory coupled to critical fermions

Chern-Simons theory coupled to massless critical fermions in the fundamental representation can be defined as a deformation of regular fermion theory [Eq. (3.10)]. The action is given by

$$
S=S_{\mathrm{CS}}+\int d^{3} x \bar{\psi} \gamma^{\mu} D_{\mu} \psi+\int d^{3} x\left(B \bar{\psi} \psi+\frac{N}{6} \lambda_{6}^{f} B^{3}\right),
$$

where $B$ is a Lagrange multiplier field and $\lambda_{6}^{f}$ is a marginal coupling in the critical fermion theory. The effective potential of the theory [29] can be written as

$$
\begin{aligned}
v[U]= & -\frac{N}{6 \pi \lambda} \tilde{c}^{3}\left(1-\lambda+\hat{g}\left(\lambda, \lambda_{6}^{f}\right)\right) \\
& -\frac{1}{2 \pi} \sum_{n=1}^{\infty} \frac{\beta_{n}}{n}\left(\operatorname{Tr} U^{n}+\operatorname{Tr} U^{\dagger n}\right),
\end{aligned}
$$

where

$$
\beta_{n}=\frac{(-1)^{n+1}}{n^{2}}(1+n \tilde{c}) e^{-n \tilde{c}} .
$$

$\tilde{c}$ is obtained by extremizing the effective potential

$$
\tilde{c}\left(\frac{1}{\lambda}-1+\frac{\hat{g}\left(\lambda, \lambda_{6}^{f}\right)}{\lambda}\right)=-\frac{1}{N} \sum_{n=1}^{\infty} \frac{(-1)^{n} e^{-n \tilde{c}}}{n}\left(\rho_{n}+\rho_{-n}\right) .
$$

Thus, in all these examples we see that the partition functions take a generic form 


$$
\mathcal{Z}_{c s}^{\text {matter }}=\left\langle e^{v[U]}\right\rangle
$$

where $v(U)=N \sum_{n=1}^{\infty} \frac{\beta_{n}}{n}\left(\operatorname{Tr} U^{n}+\operatorname{Tr} U^{\dagger n}\right)+\beta_{0}$.

This way of writing the partition function is helpful in expressing the same in terms of modular transform matrices of $u(N)_{k}$ representations of the dual WZW model.

\section{A. Connection with the WZW model}

From the examples discussed above, we see that coupling with fundamental matter fields renders an effective action of the form

$$
v(U)=N \sum_{n=1}^{\infty} \frac{\beta_{n}}{n}\left(\operatorname{Tr} U^{n}+\operatorname{Tr} U^{\dagger n}\right)+\beta_{0},
$$

where $\beta_{n}$ depends on the type of matter fields. Therefore, the partition function of $U(N)$ Chern-Simons theory of level $k$ coupled with fundamental matter can be written in general as

$$
\mathcal{Z}_{\mathrm{CS}}^{\text {matter }}\left[S^{2} \times S^{1}, U(N), k\right]=\left\langle e^{N \sum_{n=1}^{\infty} \frac{\beta_{n}}{n}\left(\operatorname{Tr} U^{n}+\operatorname{Tr} U^{\dagger n}\right)}\right\rangle_{N, k} .
$$

Before we write down the partition function in terms of quantities of the $u(N)_{k}$ WZW model, we make a justified approximation in $v(U)$. Since $\beta_{n}$ s are exponentially suppressed by a factor $e^{-\sigma n}$, we truncate the sum over $n$ and approximate that the sum runs from 1 to $L$ where $L \leq k, N$. This enables us to write down the partition function in terms of modular transform matrices of the $u(N)_{k}$ WZW model. However, in the limit $k \rightarrow \infty$ and $N \rightarrow \infty$ we can give up this approximation.

Expanding the exponential we can write

$$
\mathcal{Z}_{\mathrm{CS}}^{\text {matter }}\left[S^{2} \times S^{1}, U(N), k\right]=\sum_{\vec{k}, \vec{l}} g_{\vec{k}} g_{\vec{l}}\left\langle\Upsilon_{\vec{k}}(U) \Upsilon_{\vec{l}}\left(U^{\dagger}\right)\right\rangle_{N, k},
$$

where $\vec{\beta}=\left\{\beta_{1}, \beta_{2}, \ldots, \beta_{L}\right\}$ is the set of $L$ parameters of the theory, and $\vec{k}=\left\{k_{1}, k_{2}, \ldots, k_{L}\right\}$ and $\vec{l}=\left\{l_{1}, l_{2}, \ldots, l_{L}\right\}$ are $L$-dimensional vectors with $k_{n}, l_{n} \in[0,1,2, \ldots]$. Functions $g_{\vec{k}}$ and $\Upsilon_{\vec{k}}(U)$ are given by

$$
g_{\vec{k}}=\prod_{n=1}^{L} \frac{N^{k_{n}} \beta_{n}^{k_{n}}}{n^{k_{n}} k_{n} !}, \quad \Upsilon_{\vec{k}}(U)=\prod_{n=1}^{L}\left(\operatorname{Tr} U^{n}\right)^{k_{n}} .
$$

Using the group theory identity one can write

$$
\operatorname{Tr} U^{m}=\sum_{p=1}^{\min (m, N)}(-1)^{p-1} \operatorname{Tr}_{R_{p}} U
$$

where the index $p$ in the representation $R_{p}$ defines different higher dimensional representations in terms of Dynkin indices as

$$
R_{p}= \begin{cases}{[\underbrace{m, 0, \ldots, 0]}_{p}} & \text { for } p=1 \\ {[m-N, 0, \ldots, 0]} & \text { for } p=2, \ldots, \min (m, N-1) \\ {[m, 0, \ldots, 0,1,0,0]} & \text { for } p=N, m \geq N .\end{cases}
$$

Since $n \leq k$, all the representations $R_{p}$ are integrable representations of $u(N)_{k}$. Clubbing up everything we get

$$
\begin{aligned}
\mathcal{Z}_{\mathrm{CS}}^{\text {matter }}= & \sum_{\vec{k}, \vec{l}} g_{\vec{k}} g_{\vec{l}} \prod_{\substack{n, m \\
=1}}^{L} \sum_{\substack{r_{1}, \ldots, r_{n} \\
s_{1}, \ldots, s_{m}}} \frac{k_{n} !}{r_{1} ! \ldots r_{n} !} \frac{l_{m} !}{s_{1} ! \ldots s_{m} !}(-1)^{r_{2}+2 r_{3}+\cdots+(n-1) r_{n}+s_{2}+2 s_{3}+\cdots+(m-1) s_{m}} \\
& \left\langle\left(\operatorname{Tr}_{R_{1}} U\right)^{r_{1}} \cdots\left(\operatorname{Tr}_{R_{n}} U\right)^{r_{n}}\left(\operatorname{Tr}_{R_{1}} U^{\dagger}\right)^{s_{1}} \cdots\left(\operatorname{Tr}_{R_{m}} U^{\dagger}\right)^{s_{m}}\right\rangle_{N, k} .
\end{aligned}
$$

Using the definition (2.1) we finally write

$$
\mathcal{Z}_{\mathrm{CS}}^{\text {matter }}\left[S^{2} \times S^{1}, U(N), k\right]=\sum_{\mathcal{R}} \mathcal{S}_{0 \mathcal{R}}^{2} \exp \left[N \sum_{n=1}^{L} \frac{\beta_{n}}{n} \sum_{p=1}^{\min (n, N)}(-1)^{p-1}\left(\frac{\mathcal{S}_{\mathcal{R} R_{p}}+\mathcal{S}_{\mathcal{R} \bar{R}_{p}}}{\mathcal{S}_{0 \mathcal{R}}}\right)\right] .
$$

In the limit $k, N \rightarrow \infty$ we can lift the restriction over $n$ and all the $R_{p}$ 's are integrable representations of $u(N)_{k}$. In that case, we can write the partition function of CS theory on $S^{2} \times S^{1}$ coupled with fundamental matter in terms of quantities of the related WZW model as

$$
\mathcal{Z}_{\mathrm{CS}}^{\text {matter }}\left[S^{2} \times S^{1}, U(N), k\right]=\sum_{\mathcal{R}} \mathcal{S}_{0 \mathcal{R}}^{2} \exp \left[N \sum_{n=1}^{\infty} \frac{\beta_{n}}{n} \sum_{p=1}^{\min (n, N)}(-1)^{p-1}\left(\frac{\mathcal{S}_{\mathcal{R} R_{p}}+\mathcal{S}_{\mathcal{R} \bar{R}_{p}}}{\mathcal{S}_{0 \mathcal{R}}}\right)\right]
$$


Transformation of modular transformation matrices under level-rank duality is well known [17-19,21]. It would be interesting to see the level-rank duality between CS theory coupled with different fundamental matters using the dualities of modular transformation matrices.

\section{B. Gross-Witten-Wadia potential-a toy model}

Now we consider toy examples and discuss the dominant large $N$ representations corresponding to different phases of the theory. We also show how different large $N$ representations are related to each other by transposition as a consequence of level-rank duality.

We consider Chern-Simons theory on $S^{2} \times S^{1}$ coupled with GWW potential. The partition function is given by

$$
\mathcal{Z}_{\mathrm{CS}}^{\mathrm{GWW}}=\int[\mathcal{D} A] e^{S_{\mathrm{CS}}+N \beta_{1}\left(\operatorname{Tr} U+\operatorname{Tr} U^{\dagger}\right)}=\left\langle e^{N \beta_{1}\left(\operatorname{Tr} U+\operatorname{Tr} U^{\dagger}\right)}\right\rangle_{N, k} .
$$

This particular example is self-dual, since under level-rank duality the GWW potential transforms into itself [16]. Using the relation (3.30) one can write the above partition function in terms of modular transform matrices

$$
\mathcal{Z}_{\mathrm{CS}}^{\mathrm{GWW}}=\sum_{\mathcal{R}} \mathcal{S}_{0 \mathcal{R}}^{2} e^{N \beta_{1}\left(\frac{\mathcal{S}_{\mathcal{R F}}+\mathcal{S}_{\mathcal{R} \overline{\mathcal{F}}}}{s_{0 \mathcal{R}}}\right)}
$$

Here, $\mathcal{F}$ and $\overline{\mathcal{F}}$ stand for fundamental and antifundamental representations. In the large $N$ limit one can easily calculate that

$\frac{\mathcal{S}_{\mathcal{R} \mathcal{F}}}{\mathcal{S}_{\mathcal{R} 0}}=e^{-2 \pi i \phi} \sum_{i=1}^{N} e^{\frac{2 \pi i}{N+k} h_{i}} \quad$ and $\quad \frac{\mathcal{S}_{\mathcal{R} \overline{\mathcal{F}}}}{\mathcal{S}_{\mathcal{R} 0}}=e^{2 \pi i \bar{\phi}} \sum_{i=1}^{N} e^{-\frac{2 \pi i}{N+k} h_{i}}$,

where $\phi=\frac{Q(R)(Q(\mathcal{F})-Q(0))+s^{R}}{N(k+N)}$ and $\bar{\phi}=\frac{Q(\mathcal{R})(Q(\mathcal{F})-Q(0))+s^{R}}{N(k+N)}$. By appropriately choosing the eigenvalues of $u(1)$ generators $Q$ 's, we can set $\phi=0$ and $\bar{\phi}=0$. Hence the partition function is given by

$$
\begin{aligned}
\mathcal{Z}_{\mathrm{CS}}^{\mathrm{GWW}}= & 2^{N(N-1)}\left(\frac{1}{k+N}\right)^{N} \\
& \times \sum_{\vec{h}} \prod_{i<j} \sin ^{2}\left(\frac{\pi\left(h_{i}-h_{j}\right)}{k+N}\right) e^{2 N \beta_{1} \sum_{i=1}^{N} \cos \left(\frac{2 \pi}{N+k} h_{i}\right)} .
\end{aligned}
$$

In the large $N$ limit we define

$$
h(x)=\frac{h_{i}}{N}, \quad \text { where } x=\frac{i}{N}, \quad x \in[0,1],
$$

with

$$
0 \leq h(x) \leq \frac{1}{\lambda}
$$

The partition function, in this limit, is given by

$$
\mathcal{Z}_{\mathrm{CS}}^{\mathrm{GWW}}=\mathcal{A} \int[d h] e^{-N^{2} S_{\mathrm{eff}}[h(x)]},
$$

where

$$
\begin{aligned}
S_{\mathrm{eff}}[h(x)],= & -f d x d y \ln |\sin (\pi \lambda(h(x)-h(y)))| \\
& -2 \beta_{1} \int d x \cos (2 \pi \lambda h(x)) .
\end{aligned}
$$

Redefining the variable $h(x)$,

$$
\theta(x)=2 \pi \lambda h(x), \quad \text { with } \quad 0 \leq \theta(x) \leq 2 \pi,
$$

the effective action in terms of $\theta(x)$ can be written as

$$
\begin{aligned}
S_{\text {eff }}[\theta(x)],= & -f d x d y \ln \left|\sin \left(\frac{\theta(x)-\theta(y)}{2}\right)\right| \\
& -2 \beta_{1} \int d x \cos \theta(x) .
\end{aligned}
$$

The saddle point equation in terms of eigenvalue density [Eq. (2.13)] is given by

$$
f d \theta^{\prime} \rho\left(\theta^{\prime}\right) \cot \frac{\theta-\theta^{\prime}}{2}-2 \beta_{1} \sin \theta=0 .
$$

To find different large $N$ representations one has to solve this equation with the constraint $\rho(\theta) \leq \frac{1}{2 \pi \lambda}$. This equation is exactly the same as the eigenvalue equation discussed in [16]. Therefore, in the large $N$ limit, the dominant representations are completely determined by the corresponding dominant eigenvalue distributions studied in [16].

\section{Large $N$ representations}

In this section we study the dominant large $N$ integrable representations of $u(N)_{k}$ corresponding to Chern-Simons theory coupled with Gross-Witten-Wadia potential.

No-gap solution.-The no-gap phase for the capped GWW model is identical with that of the uncapped model. Eigenvalue distribution is given by

$$
\rho(\theta)=\frac{1}{2 \pi}\left(1+2 \beta_{1} \cos \theta\right) .
$$

$\rho(\theta)$ is maximum (minimum) at $\theta=0,2 \pi \quad(=\pi)$. Therefore, from (2.14), we find that the no-gap phase is valid for 


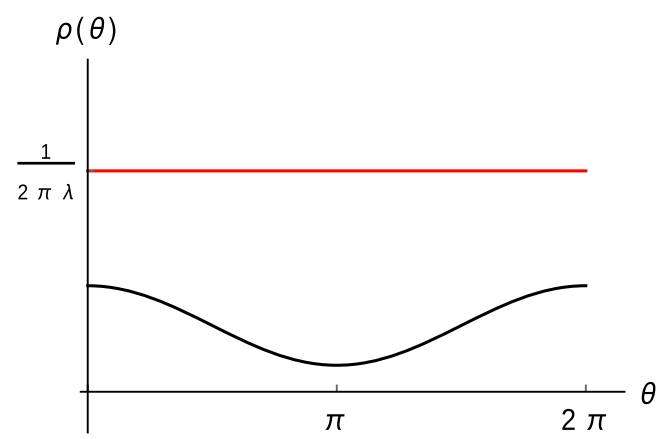

(a)

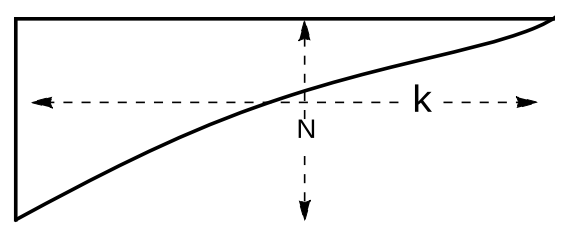

(b)

FIG. 1. Eigenvalue distribution and the corresponding dominant Young diagram for the no-gap phase. (a) $\rho(\theta)$ vs $\theta$ for no-gap phase (black curve). The red line denotes the upper-cap. (b) A typical Young diagram for no-gap phase. There are maximum $\kappa$ columns. Young distribution function never saturates the upper bound 1 .

$$
\begin{aligned}
& \beta_{1}<\frac{1}{2 \lambda}-\frac{1}{2} \quad \text { for } \lambda>\frac{1}{2} \\
& \beta_{1}<\frac{1}{2} \quad \text { for } \lambda<\frac{1}{2} .
\end{aligned}
$$

In Fig. 1 we plot the eigenvalue density for $\left(\beta_{1}, \lambda\right)$ in the range mentioned in (3.44).

Lower-gap solution.-Eigenvalue distribution for this phase is also the same as the one-gap solution for the uncapped GWW model [12],

$\rho(\theta)=\frac{2 \beta_{1}}{\pi} \sqrt{\frac{1}{2 \beta_{1}}-\sin ^{2} \frac{\theta}{2}}\left|\cos \frac{\theta}{2}\right|, \quad$ for $\sin ^{2} \frac{\theta}{2}<\frac{1}{2 \beta_{1}}$

$\rho(\theta)=0 . \quad$ for $\sin ^{2} \frac{\theta}{2}>\frac{1}{2 \beta_{1}}$.

The gap and distribution are distributed symmetrically around $\pi$. The maximum of this distribution is again at $\theta=0$. This phase only exists for $\beta_{1} \geq \frac{1}{2}$. Now we have further restriction due to upper limit of $\rho(\theta)$, which implies

$$
\beta_{1} \leq \frac{1}{8 \lambda^{2}}
$$

Thus, a lower-gap solution exists for

$$
\beta_{1}<\frac{1}{8 \lambda^{2}} \text { and } \quad \lambda \leq \frac{1}{2}
$$

For $\lambda>\frac{1}{2}$ this solution does not exist.

In Fig. 2 we plot the eigenvalue density for $\left(\beta_{1}, \lambda\right)$ in the range mentioned in (3.44). $\rho(\theta)=0$ [equivalently $u(h)=0$ ] implies that there is a horizontal jump in the Young diagram (blue line in the figure).

Upper-cap solution.-This is the first new phase in the capped GWW matrix model as well as any capped matrix

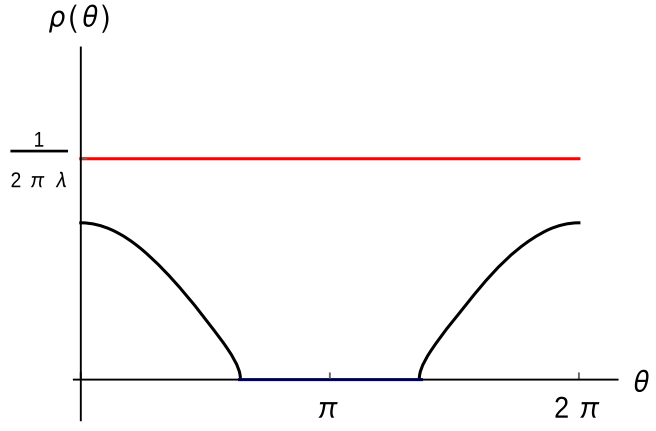

(a)

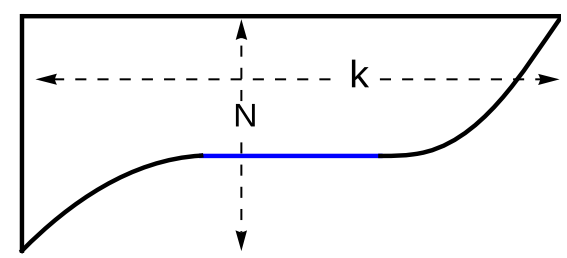

(b)

FIG. 2. Eigenvalue distribution and the corresponding dominant Young diagram for the lower-gap phase. (a) $\rho(\theta)$ vs $\theta$ for lower-gap phase (black curve). The red line denotes the upper-cap. The blue line corresponds to gap in eigenvalue distribution. (b) A typical Young diagram for lower-gap phase. There are maximum $\kappa$ columns. $\rho(\theta)=0$ ) (equivalently $u(h)=0$ ) implies there is a horizontal jump in the Young diagram (blue line in the gure). Young distribution function never saturates the upper bound 1. 


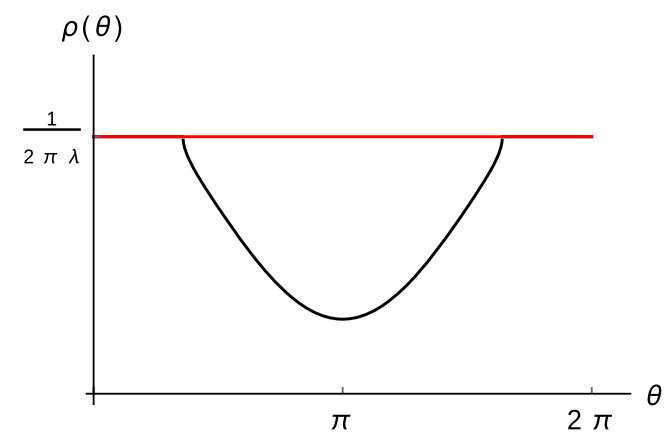

(a)

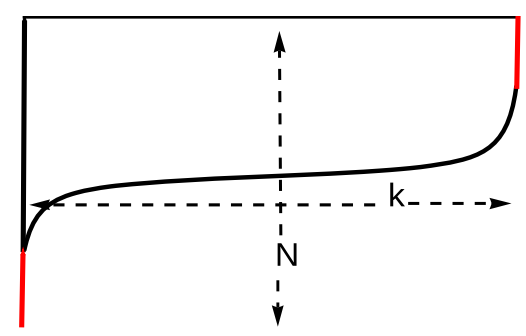

(b)

FIG. 3. Eigenvalue distribution and the corresponding dominant Young diagram for the upper-cap phase. (a) $\rho(\theta)$ vs $\theta$ for upper-cap phase (black curve). The red line denotes the upper-cap. (b) A typical Young diagram for upper-cap phase. There are maximum $\kappa$ columns. Top red line corresponds to saturation of first few rows and the bottom red line implies last few rows are empty.

models. In this phase though eigenvalues are distributed like a no-gap solution distribution is saturated over some finite range. Following [16] one can find eigenvalue density for the upper cap solution as

$\rho(\theta)=\frac{1}{2 \pi \lambda}-2 \beta_{1} \frac{\left|\sin \frac{\theta}{2}\right|}{\pi} \sqrt{\frac{\frac{1}{\lambda}-1}{2 \beta_{1}}-\cos ^{2} \frac{\theta}{2}}$ for $\cos ^{2} \frac{\theta}{2}<\frac{\frac{1}{\lambda}-1}{2 \beta_{1}}$

$\rho(\theta)=\frac{1}{2 \pi \lambda}$ for $\cos ^{2} \frac{\theta}{2}>\frac{\frac{1}{\lambda}-1}{2 \beta_{1}}$.

The minimum of this solution occurs at $\theta=\pi$ with the value

$$
\frac{1}{2 \pi}\left(\frac{1}{\lambda}-2 \sqrt{2 \beta_{1}} \sqrt{\frac{1}{\lambda}-1}\right) .
$$

Now apart from being real, minimum value should also be greater than 0 . Hence, this solution exists for

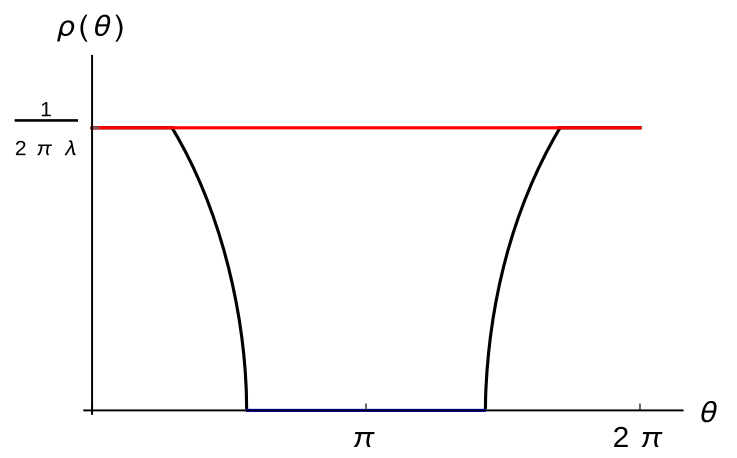

(a)

$$
\frac{1}{2 \lambda}-\frac{1}{2}<\beta_{1}<\frac{1}{8 \lambda(1-\lambda)} \quad \text { for } \lambda \geq \frac{1}{2} .
$$

The upper-cap solution does not exist for $\lambda<\frac{1}{2}$.

In Fig. 3 we see eigenvalue distribution as a function of $\theta$ and the corresponding Young distribution. The eigenvalue density touches the upper cap, i.e., $1 / 2 \pi \lambda$ in some range of $\theta$. This implies that the corresponding Young distribution function $u(h)$ touches 1 . The Young distribution function touching unity implies that a finite fraction of rows have the same box numbers. Since $h=0$ corresponds to no box in the last row, this distribution implies that a finite fraction of rows in the diagram are empty. Similarly, at the top a finite fraction of rows have $k$ boxes in each (two red lines in the Young diagram).

Upper cap with lower-gap solution.-The exact form of the solution for the upper cap with a lower gap is given in [16]. Here we plot the corresponding eigenvalue density and the associated Young diagram in Fig. 4. Here eigenvalue

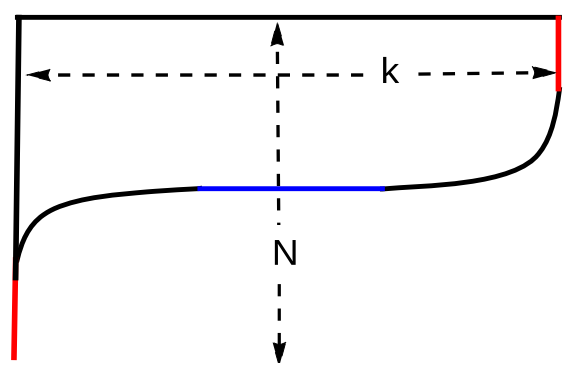

(b)

FIG. 4. Eigenvalue distribution and the corresponding dominant Young diagram for the cap-gap phase. (a) $\rho(\theta)$ vs $\theta$ for cap-gap phase (black curve). The red line denotes the upper-cap. (b) A typical Young diagram for cap-gap phase. Young diagram has a finite fraction of empty rows at the bottom and maximally saturated rows at the top. In between there are finite fraction of columns with same number of boxes. 
density touches both the upper cap $1 / 2 \pi \lambda$ and the lower bound 0 . Hence, the corresponding Young diagram has a finite fraction of empty rows at the bottom and maximally saturated rows at the top. In between there are finite fraction of columns with the same number of boxes.

\section{Level-Rank duality and transposition of diagrams}

The level-rank duality in terms of level $k$ and rank $N$ is given by $N \rightarrow k$ and $k \rightarrow N$. As a result, under level-rank duality, the 't Hooft coupling constant $\lambda$ transforms as

$$
\begin{aligned}
& \lambda^{D}=\frac{k}{k+N}=1-\lambda, \\
& \lambda^{D} \text { is the 't Hooft coupling in dual theory. }
\end{aligned}
$$

Demanding that the partition function is invariant under level-rank duality, we find that the second coupling constant $\beta_{1}$ also transforms under level-rank duality as

$\beta_{1}^{D}=\frac{\lambda}{1-\lambda} \beta_{1} ; \quad \beta_{1}^{D}$ is the coupling in dual theory.

It was shown in [16] that under level-rank duality the eigenvalue densities for lower-gap and upper-cap phase are related to each other by

$$
\tilde{\rho}(\theta)=\frac{\lambda}{1-\lambda}\left[\frac{1}{2 \pi \lambda}-\rho(\theta+\pi)\right] .
$$

From the relation between $\rho(\theta)$ and $u(h)$ [Eq. (2.16)] we see that under level-rank duality, Young distributions are related by

$$
\tilde{u}(h)=\frac{\lambda}{1-\lambda}\left[1-u\left(h+\frac{1}{2 \lambda}\right)\right] .
$$

The above relation is a two-step process. In the first step we shift the hook length by $\frac{1}{2 \lambda}(h \rightarrow h+1 / 2 \lambda)$. Since the potential is periodic we can extend $h$ beyond $1 / \lambda$ with the identification $h \sim h+1 / \lambda$. One can, therefore, periodically stack Young diagrams one after one (blue boxes) in Fig. 5. A shift of $1 / 2 \lambda$ in $h$ means we go from blue box to red box. The next step is $u(h) \rightarrow 1-u(h)$. This implies transposition of the shifted diagram; i.e., row and columns are interchanged. In Fig. 5 we start with the Young diagram for the one-gap phase (diagram 1). After giving a shift in $h$ we obtain diagram 2. Young diagram 3 is obtained from diagram 2 by transposition. Diagram 3 is the dominant one for the upper-cap phase (Fig. 3).

Since no-gap and cap-gap phases are dual to themselves, we see from the corresponding dominant Young diagrams that the above set of operations gives us back the same Young diagrams.

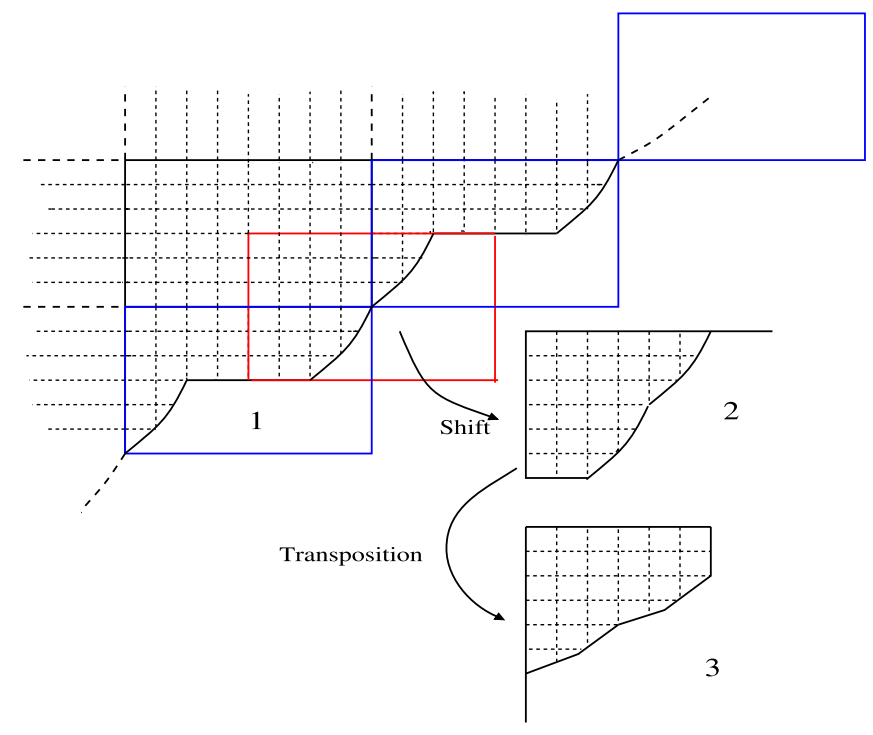

FIG. 5. Duality in Young diagrams.

\section{CHERN-SIMONS THEORY ON $S^{3}$}

A special class of Seifert manifold $(g=0, p=1)$ is three sphere: $\mathcal{M}_{0,1}=S^{3}$. Chern-Simons partition function on $S^{3}$ can be obtained from the generic expression (2.1),

$\mathcal{Z}_{\mathrm{CS}}\left[S^{3}, U(N), k\right]=\sum_{\mathcal{R}} \mathcal{S}_{0 \mathcal{R}}^{2} \mathcal{T}_{\mathcal{R} \mathcal{R}}^{-1}$, in Seifert framing.

Using the properties of modular transform matrices ${ }^{16}$ one can show that the partition function in Seifert framing is the same as that in canonical framing up to a phase factor [see Eq. (1.6)],

$$
\mathcal{Z}_{\mathrm{CS}}\left[S^{3}, U(N), k\right]=\mathcal{S}_{00} \text { in canonical framing. }
$$

The partition function in Seifert framing can be written as a matrix model. In the large $N$ limit we see that one integrable representation (say $\tilde{\mathcal{R}}$ ) dominates the partition function and the value of the partition function for that representation is the same as $\mathcal{S}_{00}$,

$$
\mathcal{Z}_{\mathrm{CS}}\left[S^{3}, U(N), k\right]=\lim _{N \rightarrow \infty} \mathcal{S}_{0 \tilde{\mathcal{R}}}^{2} \mathcal{T}_{\tilde{\mathcal{R}} \tilde{\mathcal{R}}}^{-1}=\mathcal{S}_{00}
$$

Using the expressions for modular transform matrices in terms of Young diagram data, the partition function for Chern-Simons theory on $S^{3}$ can be written as a matrix model, ${ }^{17}$

\footnotetext{
${ }^{16} S^{2}=1,(S T)^{3}=S^{2}=1$.

${ }^{17}$ We appropriately choose $u(1)$ eigenvalues such that in the large $N$ limit the partition function matches that in canonical framing (4.2).
} 


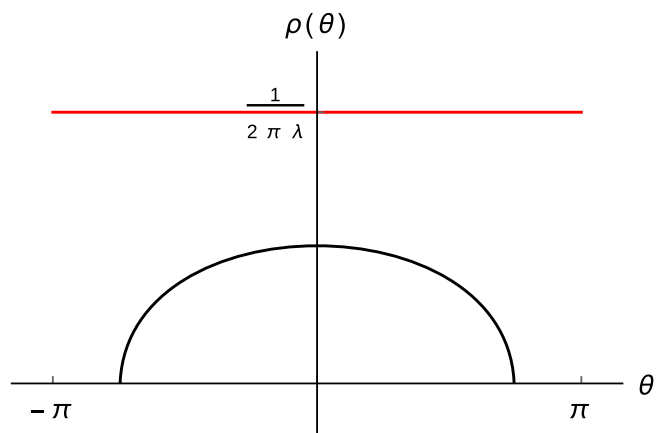

(a)

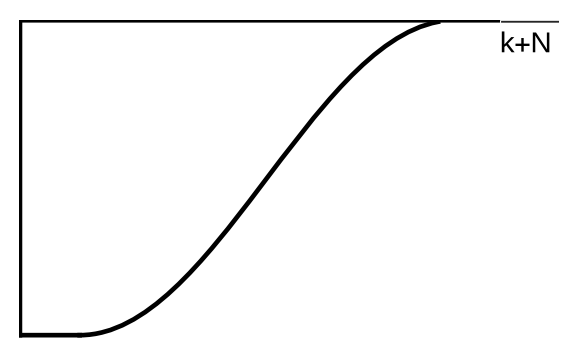

(b)

FIG. 6. Eigenvalue distribution and the corresponding dominant Young diagram for the one-gap phase for CS theory on $S^{3}$. (a) $\rho(\theta)$ vs $\theta$ for one-gap phase (black curve). The red line denotes the upper-cap. (b) A typical Young diagram for one-gap phase.

$$
\begin{aligned}
\mathcal{Z}_{\mathrm{CS}}\left[S^{3}, U(N), k\right]= & \left(\frac{2^{(N-1)}}{k+N}\right)^{N} e^{-\frac{i \pi g_{s}\left(N^{3}-N\right)}{6}} \\
& \times \sum_{\vec{h}} \prod_{i<j} \sin ^{2}\left[\pi g_{s}\left(h_{i}-h_{j}\right)\right] e^{-i \pi g_{s}} \\
\text { where, } g_{s}= & \frac{1}{k+N} \text { is coupling constant and } \\
\Delta & =\frac{1}{2}(N-1-2 s) .
\end{aligned}
$$$$
\times \sum_{\vec{h}} \prod_{i<j} \sin ^{2}\left[\pi g_{s}\left(h_{i}-h_{j}\right)\right] e^{-i \pi g_{s} \sum_{i}\left(h_{i}-\Delta\right)^{2}}
$$

There are two possible ways to deal with this partition function. Replacing $g_{s} \rightarrow-i g_{s}{ }^{18}$ one can write this partition function as a Hermitian matrix model. This was studied by $[30,31]$ in the context of two-dimensional Yang-Mills theory. ${ }^{19}$ We briefly review the analysis in Appendix. Here, we use the similar technique (Sec. II A) to write the partition function as a unitary matrix model $[11,14]$. We take $\Delta=\frac{k+N}{2}$ with appropriate choice of $s$ and define the angular variables $\theta_{i}$,

$$
\theta_{i}=\frac{2 \pi h_{i}}{k+N}-\pi, \quad \theta_{N} \geq-\pi \quad \text { and } \quad \theta_{1} \leq \pi
$$

The summand in Eq. (4.4) can be written as a measure of $S U(N)$ (or $U(N)$ ), as described in Sec. II, with the fact that eigenvalues are now discrete. Discreteness in eigenvalues implies an upper cap in eigenvalue distribution function given by Eq. (2.14).

The partition function in the large $N$ limit after a wick rotation in $g_{s}\left(g_{s} \rightarrow i g_{s}\right)$ is given by

\footnotetext{
${ }^{18}$ This can be done by $g_{s} \rightarrow g_{s}(1+i \epsilon)$. The imaginary part creates a large damping in the large $N$ limit and the partition function is dominated by classical configuration. One can also rotate the $g_{s}$ axis by $\pi / 2$, which is equivalent to $g_{s} \rightarrow g_{s}^{\prime}=i g_{s}$ to get the dominant contribution from classical configuration. The free energy is therefore a function of $g_{s}^{\prime}=-i g_{s}$, which matches with the free energy of CS theory at large $N$ (genus 0 ).

${ }^{19}$ Also look at [5] for a review.
}

$$
\begin{aligned}
\mathcal{Z}_{\mathrm{CS}} & {\left[S^{3}, U(N), k\right] } \\
& =e^{\frac{\pi g_{S} N^{3}}{6}} \int \prod_{i} \frac{d \theta_{i}}{2 \pi} \prod_{i<j} 2 \sin ^{2}\left(\frac{\theta_{i}-\theta_{j}}{2}\right) e^{-\frac{1}{4 \pi g_{s}} \sum_{i=1}^{N} \theta_{i}^{2}} .
\end{aligned}
$$

The potential is not periodic in this case. However, to write a unitary matrix model for Chern-Simons theory we introduce periodicity in $h_{i}: h_{i} \sim h_{i}+k+N$. This implies that the harmonic oscillator potential $\theta^{2}$ is repeated beyond $-\pi \leq \theta \leq \pi$. Writing the partition function as a unitary matrix model has an advantage. In the next section (Sec. IV A) we see that at large $N$, the model also admits a two-gap solution with the same free energy. Finding this new phase is easy in the unitary matrix model.

In the large $N$ limit the eigenvalue distribution is governed by the saddle point equation,

$$
f d \theta^{\prime} \rho(\theta) \cot \left(\frac{\theta-\theta^{\prime}}{2}\right)=\frac{1}{2 \pi \lambda} \theta .
$$

This unitary matrix model was studied in [14]. It was observed that the system has only one phase in the large $N$ limit and the eigenvalue distribution was given by

$$
\rho(\theta)=\frac{1}{2 \pi^{2} \lambda} \tanh ^{-1}\left[\sqrt{1-\frac{e^{-2 \pi \lambda}}{\cos ^{2} \frac{\theta}{2}}}\right] .
$$

Since $\rho(\theta) \geq 0$, this implies that eigenvalues are distributed for $\theta \in\left[-2 \cos ^{-1} e^{-\pi \lambda}, 2 \cos ^{-1} e^{-\pi \lambda}\right]$. The eigenvalue distribution and corresponding Young diagram distribution are plotted in Fig. 6. We call this phase a one-gap phase.

As discussed in [14] the theory does not admit any nogap phase as the eigenvalue distribution for such a phase becomes negative in some range of $\theta$. No phase transition in this theory was considered in [14] because eigenvalue density was unrestricted. 
One can compute the free energy corresponding to this phase. The free energy is given by

$$
F_{\mathrm{CS}}(\lambda)=\frac{2 \pi^{3} \lambda^{3}}{3}-\frac{\pi^{3} \lambda}{3}-L i_{3}\left(e^{-2 \pi \lambda}\right)+\zeta_{3}
$$

for all $0 \leq \lambda \leq 1$. This is in agreement with $[5,23,32,33]$. This is, as expected, equal to the partition function (4.1) in canonical framing (4.2).

Interestingly, the eigenvalue distribution (4.8) is the functional inverse of the Young diagram distribution obtained in $[5,30]$ (see Appendix for details). This identification is similar to that obtained in the context of a generic unitary matrix model considered in a series of papers $[14,34,35]$. However, in this case both the distributions correspond to large $N$ integrable representation of $u(N)_{k}$. This is similar to [36].

From eigenvalue distribution (4.8), we see that the eigenvalue density saturates the upper bound at $\lambda^{*}=$ $\frac{1}{\pi} \ln \cosh \pi$. Saturation of eigenvalue density to its upper bound is equivalent to the dominant representation in the dual description, saturating the integrability condition. Therefore, one naturally expects that the system will undergo a phase transition from a gap phase to cap-gap phase after this value of $\lambda$ similar to [16]. However, we explicitly check that such cap-gap phase does not exists for CS theory of $S^{3}$, which is manifest from the partition function written in canonical frame (no such phase transition exists in canonical frame). This essentially means that the representation $\tilde{\mathcal{R}}$ that dominates the partition function in the large $N$ limit is not an integrable representation anymore. One needs to continue the sum over $\mathcal{R}$ beyond integrable representations for $\lambda>\lambda^{*}$ $[37,38]$ using the symmetries of the summand.

\section{A. Two-gap phase in Chern-Simons theory on $S^{\mathbf{3}}$}

Chern-Simons theory on $S^{3}$ does not allow any no-gap solution ${ }^{20}$ but admits multigap (more than one) solutions $[14,39,40]$. A two-gap solution, in particular, was explicitly studied in [14]. The solution was given by

$$
\begin{aligned}
\rho(\theta) & =\frac{1}{4 \pi^{2} \lambda} \tanh ^{-1}\left[\frac{\sqrt{(2 \cos \theta+\gamma)^{2}-4 e^{-4 \pi \lambda}}}{2 \cos \theta+\gamma}\right] \\
& =\frac{1}{4 \pi^{2} \lambda} \tanh ^{-1}\left[\frac{4 \sqrt{\left(\cos ^{2} \theta / 2-\cos ^{2} \theta_{1} / 2\right)\left(\cos ^{2} \theta / 2-\cos ^{2} \theta_{2} / 2\right)}}{2 \cos \theta+\gamma}\right],
\end{aligned}
$$

where

$$
\begin{aligned}
& \theta_{1}=2 \cos ^{-1}\left[\frac{\sqrt{2\left(1+e^{-2 \pi \lambda}\right)-\gamma}}{2}\right], \\
& \theta_{2}=2 \cos ^{-1}\left[\frac{\sqrt{2\left(1-e^{-2 \pi \lambda}\right)-\gamma}}{2}\right] .
\end{aligned}
$$

The parameter $\gamma$ cannot be fixed from the analyticity or normalization conditions of the resolvent. Hence it is a one parameter family of solutions at this point. However, the free energy for the two-gap phase depends on the parameter $\gamma$ and, hence, it is possible to cook up a new large $N$ solution or phase for a given value of $\lambda$ such that the free energy of that phase exactly matches with the free energy of the one-gap phase. The additional parameter $\gamma$ depends on the 't Hooft coupling $\lambda$. Since the free energy matching condition is hard to track analytically we used numerical methods to find two-gap solutions with the same free energy as of the one-gap solution for a given value of $\lambda$.

\footnotetext{
${ }^{20}$ The saddle point equation does not allow a real semidefinite positive eigenvalue distribution for this case.
}

The two-gap eigenvalue density (4.10) is defined only when $\theta \in\left(-\pi,-\theta_{1}\right\} \cup\left\{-\theta_{2}, \theta_{2}\right\} \cup\left\{\theta_{1}, \pi\right\}$; therefore, it is quite clear if $\theta_{1}=\pi$ then the two-gap solution should smoothly go to the one-gap solution and the free energies should match. This simple observation validates our numerical observations as well. One can observe from Fig. 7(a) (lower graph) that the analytic prediction (continuous line) indeed matches with the numerical prediction (discrete points in the figure). For all points on this line, the free energy of two-gap solution trivially matches with that of the one-gap phase.

In our numerical analysis we also find some nontrivial solutions (both $\theta_{1}$ and $\theta_{2}$ real, different and between 0 and $\pi$ ) of $\gamma$ as well for which the free energy matches with (4.9). We find that the nontrivial two-gap solution exists for $\lambda$ greater than a minimum value of $\lambda_{0}=0.3545 \pm .0045$. All the black points in Fig. 7(a) (upper graph) generate a two-gap solution for which the free energy matches with the one-gap case. Surprisingly we observe that for $\lambda>\lambda^{*}$ this two-gap solution is also plagued with the same pathology, i.e., eigenvalue density exceeding the value of the upper cap. For all the red points marked in the upper graph in Fig. 7(a), one can generate a two-gap solution but for all of those points eigenvalue density goes beyond the capped value. In Fig. 8 we plot eigenvalue distribution for the one-gap and 


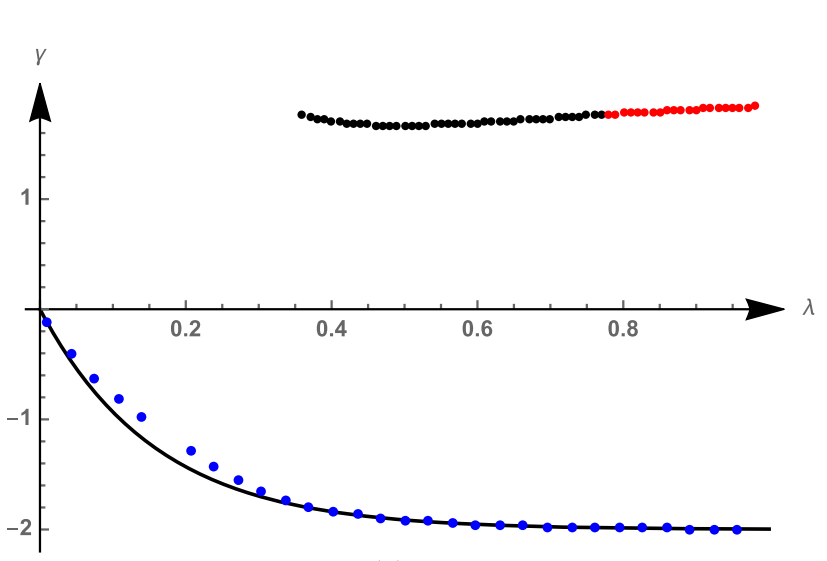

(a)

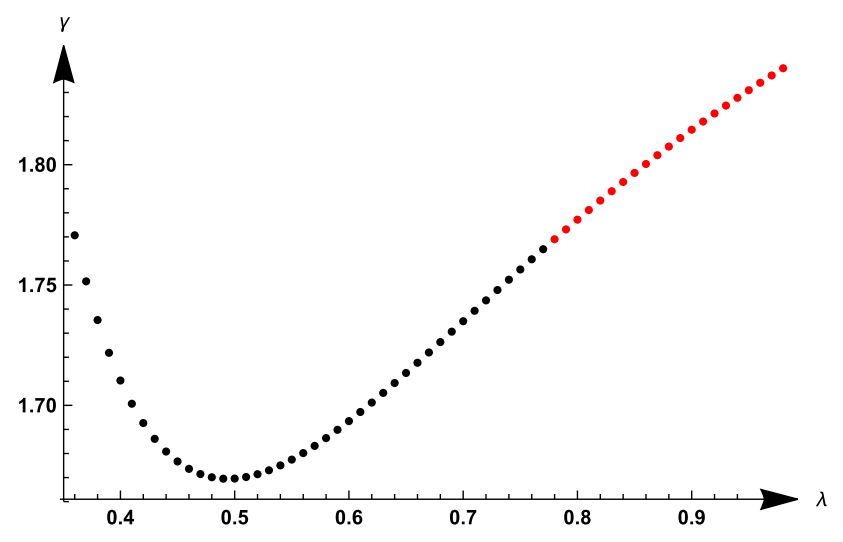

(b)

FIG. 7. Equienergy plot of $\gamma$ vs $\lambda$. Continuous lines denote the analytic part and discrete points are the numerical findings. (a) Numerical equi-energy plot for $\gamma$ vs $\lambda$. (b) Zoomed in plot of $\gamma$ vs $\lambda$ for two-gap phase (the upper graph of 7a)

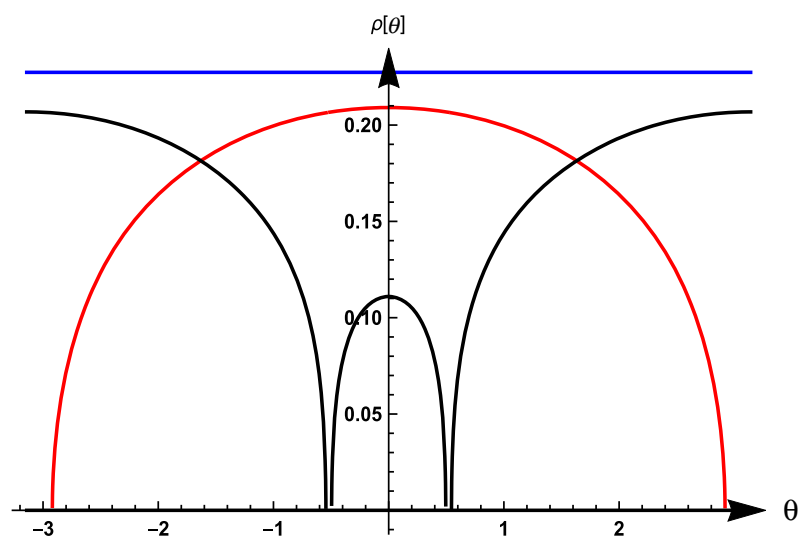

(a)

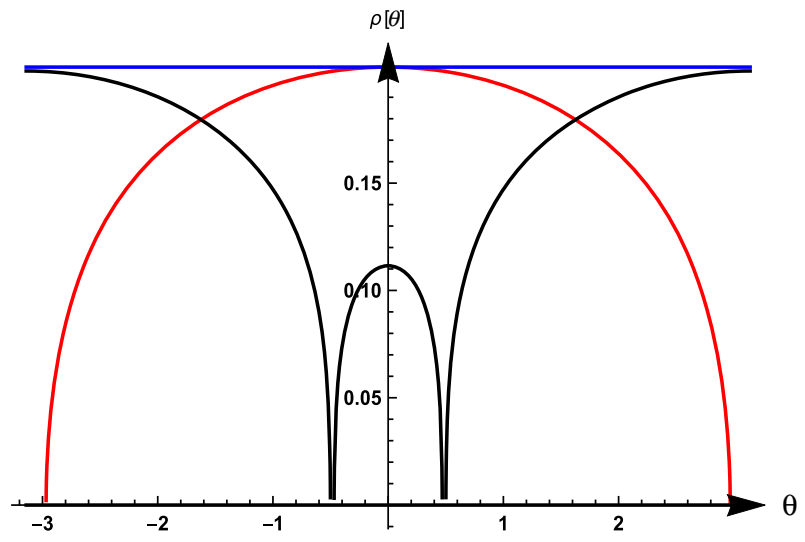

(b)

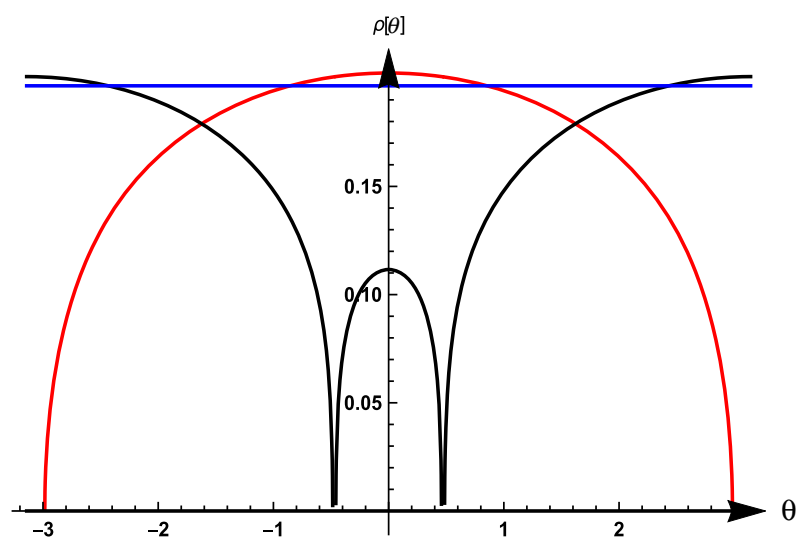

(c)

FIG. 8. $\rho(\theta)$ vs $\theta$ for the one-gap phase (red curve), two-gap phase (black curve), and upper saturation limit (blue line) for CS theory on $S^{3}$. (a) for $\lambda=0.7$ and $\gamma=1.73496$. (b) for $\lambda=\lambda *$ and $\gamma=1.76900$. (c) for $\lambda=0.81$ and $\gamma=1.78115$.

two-gap phase. For $\lambda>\lambda^{*}$, we see both one-gap and twogap solutions go beyond the saturation limit.

However, we are not very confident about the minimum value of $\lambda$, i.e., $\lambda_{0}$. Numerically we were not able to find any real acceptable values of $\theta_{1}$ and $\theta_{2}$ below $\lambda_{0}$ such that the free energy matches with the one-gap phase. This could be because of our lack of expertise in numerical analysis. 
One of the primary goals of this numerical exercise is to establish the fact that, in principle, one can find two-gap solutions ${ }^{21}$ for CS theory on $S^{3}$, which has the same free energy as the one-gap phase. It should be emphasized that our objective in this section is to demonstrate the existence of multigapped phases in the matrix model side, not to study any phase transition. As of now, these two different distributions mean two different integral representations for the observables of the theory. Although the underlying meaning of these multigap phases (condensation of $D 2$ brane instantons? [40]) is not very clear yet, we attempt to show that such phases can be engineered with free energy the same as that of the one-gap phase. Understanding the physical meaning of these multigap phases (in the topological string theory side) is an interesting avenue to pursue.

\section{CONCLUSION}

In this paper we deal with a direct way of rewriting the partition functions of $S U(N)$ Chern-Simons theories on Seifert three manifolds as unitary matrix models and study the phase structure in the large $N$ limit. We start by considering the relation between the expectation value of Wilson loops and modular transformation matrices of affine lie algebra pointed out by [4]. Depending on a choice of framing one can write the partitions functions in several possible equivalent ways. We chose a particular framing called the Seifert framing. In this particular choice of framing Chern-Simons partition functions can be written as functions of modular transformation matrices of the corresponding affine lie algebra of the WZW model summed over the highest weight representations. We show that by expressing the modular transformation matrices in terms of hook numbers of the corresponding integrable representations one can recover the unitary matrix models discussed in the literature of Chern-Simons theories on Seifert manifolds [11,30,37].

Our procedure naturally explains one crucial property that has been observed in Chern-Simons theories on $S^{2} \times S^{1}$ coupled with matter in the fundamental representation [16]. It was observed in [16] that, by carefully making correct gauge choices, one can write the full partition function as a UMM where eigenvalues of unitary matrices are discrete. In this paper we have seen that the discreteness in eigenvalues is universal for Chern-Simons theory on any three manifold that can be reached by doing surgery on $S^{2} \times S^{1}$. Here also we come across the notion of Young diagram density much in line with our previous works [14,15,34,35], but it should be noted that the Young diagram densities observed in this paper are different from that one discussed in our earlier works both in origin and

\footnotetext{
${ }^{21}$ and also multigap solutions in the same spirit.
}

interpretation. In our earlier works we expressed partition function of any $U(N)$ (or $S U(N)$ ) gauge theories (ChernSimons theories, in particular) as a sum of representations of $U(N)$, where there was no restriction on the maximum number of columns. However, for Chern-Simons theory on $S^{2} \times S^{1}$, in particular, we have seen that discreteness in eigenvalue distribution put a restriction on the number of columns in $U(N)$ representations [15]. This restriction is equivalent to the integrability condition on representation of affine group $U(N)_{k}$. In fact, it was shown in [15] that the eigenvalue distribution and Young diagram distribution for Chern-Simons theory on $S^{2} \times S^{1}$ coupled with GWW are functional inverses of each other and bear a meaning of free Fermi description in the large $N$ limit. A clear hint was obtained from this work that the partition function for Chern-Simons theory on $S^{2} \times S^{1}$ has a connection with integrable representations of affine Lie group $U(N)_{k}$. Motivated by that, in this paper we start with the result of [4] and write the partition function of Chern-Simons theory on $S^{2} \times S^{1}$ coupled with GWW (this can be generalized to other fundamental matters) in terms of the unitary matrix model. To our surprise, we see that at large $N$ Young diagram distributions are similar to the eigenvalue distribution of [16] (unlike the functional inverse of the same as observed in [15]). An advantage of writing the partition function as a sum over the integrable representation is that levelrank duality is manifest in terms of transposition of Young diagrams. We check this explicitly for GWW potential. Apart from answering the origin of our previous observation in [15], this paper nicely explains the level-rank duality of Chern-Simons theories in a manifest way in a certain sense. We have shown here that thermal partition functions of CS theory coupled with regular bosons or critical Fermions on $S^{2}$ can be written as an effective single plaquette model and, in principle, one should be able to show the duality between these theories using the level-rank duality relation between modular transform matrices.

As the manifold $S^{3}$ can be constructed out of $S^{2} \times S^{1}$ by means of surgery, it is no surprise that we find the same discreteness in eigenvalues in pure Chern-Simons theory on $S^{3}$ as well. Since CS theory on $S^{3}$ is purely topological one expects that there is no phase transition as shown earlier [33,37]. Therefore, the eigenvalue density should not saturate the upper bound. But surprisingly we found that at a particular value of the 't Hooft coupling defined as $\lambda^{*}$ in this text, the eigenvalue density saturates the maximum limit. However, no phase transition was observed at this value of 't Hooft coupling. We only understand that at large $N$ the dominant representation is not integrable anymore after $\lambda>\lambda *$. Looking at the same problem through the canonical framing in place of the Seifert framing it is clear that there is no such phase transition as shown by [33]. It turns out that one can actually use the 
symmetry of the partition function to lift the constraint on the representation or in other words the constraint on the maximum number of columns in a representation and allow nonintegrable representations to dominate the partition function as well $[37,38]$.

Using our earlier result $[14]^{22}$ we discuss the existence of a two-gap phase in Chern-Simons theory on $S^{3}$ and numerically tried to look for a solution ${ }^{23}$ with the same free energy as that of the one-gap phase. But it turns out that even those solutions also saturate the upper value at $\lambda=\lambda^{*}$. We find that our two-gap solution breaks down for $\lambda$ less that a lower critical value $\lambda_{0}$. But this could be a pathology of our numerical analysis. The bottom line of our analysis is that at large $N$ there exists a two-gap phase for Chern-Simons theory on $S^{3}$ that has the same free energy as the one-gap phase for a finite range of 't Hooft coupling. This work also raises another interesting question about the existence of multigap phases for Chern-Simons theory on $S^{3}$. We are now trying to investigate the physical origin/meaning of these equal free energy multigap phases in the context of its dual topological string theory [23].

\section{ACKNOWLEDGMENTS}

We thank Rajesh Gopakumar for many helpful discussions. We are grateful to S. Minwalla, D. Ghoshal, P. Dutta, R. Loganayagam, V. Singh, D. Mukherjee, and S. Govindarajan for discussion. The work of S.D. is supported by Grant No. EMR/2016/006294 from the Department of Science and Technology, Government of India. S. D. and A. C. acknowledge the Simons Associateship of the Abdus Salam ICTP, Trieste, Italy. A. C. thanks Robert de Mello Koch and all the organizers of third Mandelstam School and Workshop at Durban, where a part of the draft was written. A. C. thanks the hospitality of ICTP, Trieste where the final draft was prepared. We are indebted to the people of India for their unconditional support toward research in basic sciences.

\section{APPENDIX: A DUAL MATRIX MODEL DESCRIPTION}

We start with Eq. (4.4). Replacing $g_{s} \rightarrow-i g_{s}$ one can write this partition function as a Hermitian matrix model.

$$
\begin{aligned}
\mathcal{Z}_{\mathrm{CS}}\left[S^{3}, U(N), k\right]= & \left(\frac{2^{(N-1)}}{k+N}\right)^{N} e^{-\frac{\pi g_{s}\left(N^{3}-N\right)}{6}} \\
& \times \sum_{\vec{h}} \prod_{i<j} \sinh ^{2}\left(\pi g_{s}\left(h_{i}-h_{j}\right)\right) e^{-\pi g_{s} \sum_{i} h_{i}^{2}} .
\end{aligned}
$$

\footnotetext{
${ }^{22}$ Supported by numerical calculations of [39].

${ }^{23}$ Though numerically challenging, but one can certainly look for such equifree energy solutions for higher gap phases as well.
}

The partition function in large $N$ limit is given by

$$
\mathcal{Z}_{c s}\left[S^{3}, U(N), k\right]=\mathcal{A}(N, k) \int[\mathcal{D} h] e^{-N^{2} S_{\text {eff }}[h]}
$$

where

$$
\begin{aligned}
S_{\mathrm{eff}}[h],= & -\int d h u(h) f d h^{\prime} u\left(h^{\prime}\right) \ln \mid \sinh \left[\pi \lambda\left(h-h^{\prime}\right] \mid\right. \\
& +\pi \lambda \int h^{2} u(h) d h .
\end{aligned}
$$

Here, $u(h)$ is the Young diagram distribution function defined in Eq. (2.15) with the constraint $u(h) \leq 1$. Although $h$ is a positive variable ranging between 0 and $1 / \lambda$, the effective action (A3) being an even function of $h$, one can extend the range of $h$ between $-1 / \lambda$ to $1 / \lambda$. In the large $N$ limit, the dominant contribution to the partition function is determined by the saddle point equation

$$
f_{-1 / \lambda}^{1 / \lambda} d h^{\prime} u\left(h^{\prime}\right) \operatorname{coth}\left(\pi \lambda\left(h-h^{\prime}\right)\right)=h .
$$

The solution of this equation is given by $[30,31]$

$$
u(h)=\frac{1}{\pi} \tan ^{-1}\left[\sqrt{\frac{e^{2 \pi \lambda}}{\cosh ^{2}(\pi \lambda h)}-1}\right]
$$

for $-a \leq h \leq a$ and 0 otherwise where $a=\frac{1}{\pi \lambda} \cosh ^{-1} e^{\pi \lambda}$. It also turns out that for this solution $u(h)<1 / 2$ for $0 \leq \lambda \leq 1$. We call this one-gap solution as $u(h)=0$ in the complement region. This distribution represents a valid integrable representation for $a<1 / \lambda$, which implies that this phase is a valid phase of the theory for $0 \leq \lambda<\lambda^{*}$, where $\lambda^{*}=\frac{1}{\pi} \ln \cosh \pi$. The Young diagram distribution for $\lambda<\lambda^{*}$ and $\lambda>\lambda^{*}$ is plotted in Fig. 9. As $\lambda$ increased beyond $\lambda^{*}$ the support of $h$ is greater than $1 / \lambda$; hence this solution does not represent a valid integrable Young diagram.

We pause and observe an interesting relation between eigenvalue distribution (4.8) and Young diagram distribution (A5) similar to [36]. They are the functional inverse of each other,

$$
u(h)=\frac{\theta}{2 \pi}, \quad 2 \rho(\theta)=\frac{h_{+}-h_{-}}{2 \pi},
$$

where $h_{ \pm}$are two roots of Eq. (A5). This identification is similar to that obtained in the context of a generic unitary matrix model considered in a series of papers $[14,34,35]$ except for an extra two factor sitting in front of $\rho(\theta)$ in the second relation. Since $h_{+}-h_{-}$has a maximum value $2 / \lambda$, the eigenvalue distribution has an upper cap $\frac{1}{2 \pi \lambda}$. All of the eigenvalue distribution with $\rho(\theta) \leq \frac{1}{2 \pi \lambda}$ 


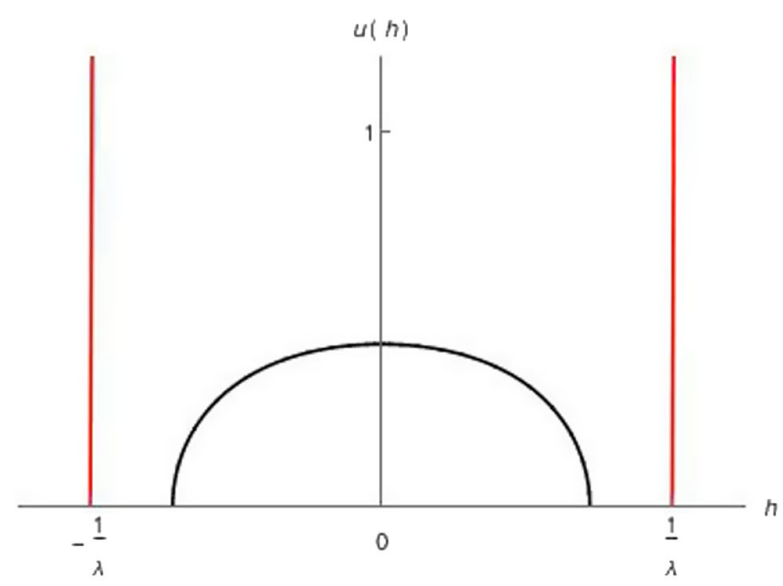

(a)

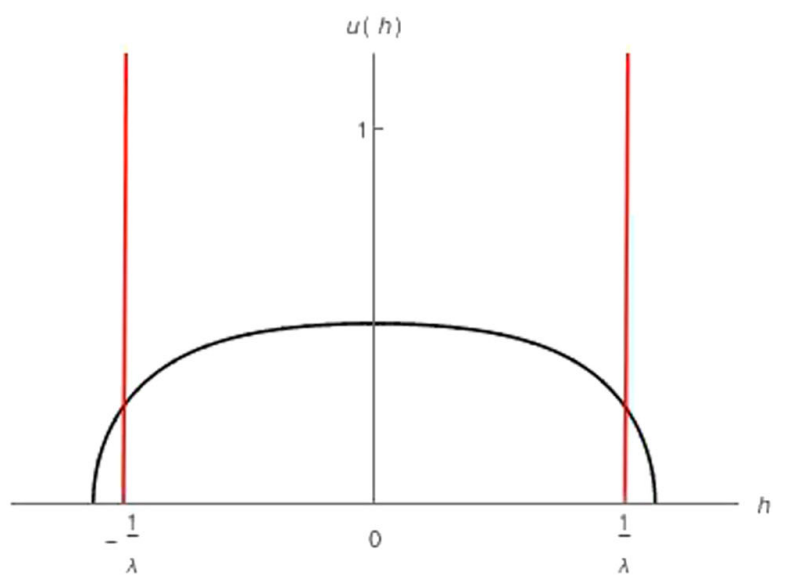

(b)

FIG. 9. $u(h)$ vs $h$ for different values of $\lambda$. (a) $u(h)$ vs $h$ for $\lambda<\lambda *$. (b) $u(h)$ vs $h$ for $\lambda>\lambda *$

corresponds to integrable representations in the WZW side. It is easy to check that $\rho(\theta)$ saturates the upper cap at $\lambda=\lambda^{*}$. For $\lambda>\lambda^{*}$ we see that $h$ goes beyond $1 / \lambda$ and hence the corresponding representation is not the integrable representation. The above identification also tells us that nonexistence of the no-gap phase in eigenvalue side [14] is consistent with the fact that $u(h)$ cannot have any capped phase [30].
[1] P. A. M. Dirac, The theory of magnetic poles, Phys. Rev. 74, 817 (1948).

[2] V. G. Turaev, The yang-baxter equation and invariants of links, Inventiones Mathematicae 92, 527 (1988).

[3] A. Tsuchiya and Y. Kanie, Vertex operators in conformal field theory on $\mathrm{p} 1$ and monodromy representations of braid group, in Conformal Field Theory and Solvable Lattice Models, edited by M. Jimbo, T. Miwa, and A. Tsuchiya (Academic Press, New York, 1988), pp. 297-372.

[4] E. Witten, Quantum field theory and the Jones polynomial, Commun. Math. Phys. 121, 351 (1989).

[5] M. Marino, Les Houches lectures on matrix models and topological strings, arXiv:hep-th/0410165.

[6] M. Atiyah, On framings of 3-manifolds, Topology 29, 1 (1990).

[7] P. Di Francesco, P. Mathieu, and D. Sénéchal, Conformal Field Theory (Springer, New York, 1997).

[8] S. Gukov, E. Martinec, G. W. Moore, and A. Strominger, Chern-Simons gauge theory and the AdS(3)/CFT(2) correspondence, edited by M. Shifman et al., From Fields to Strings Vol. 2 (World Scientific, Singapore, 2004), pp. 1606-1647.

[9] M. Blau, K. K. Mady, K. S. Narain, and G. Thompson, Chern-Simons theory on a general Seifert 3-manifold, arXiv:1812.10966.

[10] M. Aganagic, A. Klemm, M. Marino, and C. Vafa, Matrix model as a mirror of Chern-Simons theory, J. High Energy Phys. 02 (2004) 010.
[11] T. Okuda, Derivation of Calabi-Yau crystals from Chern-Simons gauge theory, J. High Energy Phys. 03 (2005) 047.

[12] D. J. Gross and E. Witten, Possible Third order phase transition in the large N Lattice gauge theory, Phys. Rev. D 21, 446 (1980).

[13] S. R. Wadia, $N=\infty$ Phase transition in a class of exactly soluble model Lattice gauge theories, Phys. Lett. 93B, 403 (1980).

[14] A. Chattopadhyay, P. Dutta, and S. Dutta, Emergent phase space description of unitary matrix model, J. High Energy Phys. 11 (2017) 186.

[15] A. Chattopadhyay, P. Dutta, and S. Dutta, From phase space to integrable representations and Level-Rank duality, J. High Energy Phys. 05 (2018) 117.

[16] S. Jain, S. Minwalla, T. Sharma, T. Takimi, S. R. Wadia, and S. Yokoyama, Phases of large $N$ vector ChernSimons theories on $S^{2} \times S^{1}$, J. High Energy Phys. 09 (2013) 009.

[17] S. G. Naculich, H. A. Riggs, and H. J. Schnitzer, Group level duality in WZW models and Chern-Simons theory, Phys. Lett. B 246, 417 (1990).

[18] E. J. Mlawer, S. G. Naculich, H. A. Riggs, and H. J. Schnitzer, Group level duality of WZW fusion coefficients and Chern-Simons link observables, Nucl. Phys. B352, 863 (1991).

[19] S. G. Naculich and H. J. Schnitzer, Duality between $\mathrm{SU}(N)-\mathrm{k}$ and $\mathrm{SU}(\mathrm{k})-N$ WZW models, Nucl. Phys. B347, 687 (1990). 
[20] T. Nakanishi and A. Tsuchiya, Level rank duality of WZW models in conformal field theory, Commun. Math. Phys. 144, 351 (1992).

[21] S. G. Naculich and H. J. Schnitzer, Level-rank duality of the U(N) WZW model, Chern-Simons theory, and 2-D qYM theory, J. High Energy Phys. 06 (2007) 023.

[22] O. Aharony, S. Giombi, G. Gur-Ari, J. Maldacena, and R. Yacoby, The thermal free energy in large N Chern-SimonsMatter theories, J. High Energy Phys. 03 (2013) 121.

[23] R. Gopakumar and C. Vafa, On the gauge theory/ geometry correspondence, Adv. Theor. Math. Phys. 3, 1415 (1999).

[24] M. Blau and G. Thompson, Derivation of the Verlinde formula from Chern-Simons theory and the G/G model, Nucl. Phys. B408, 345 (1993).

[25] E. J. Mlawer, S. G. Naculich, H. A. Riggs, and H. J. Schnitzer, Group level duality of WZW fusion coefficients and Chern-Simons link observables, Nucl. Phys. B352, 863 (1991).

[26] S. Dwivedi, V. K. Singh, S. Dhara, P. Ramadevi, Y. Zhou, and L. K. Joshi, Entanglement on linked boundaries in Chern-Simons theory with generic gauge groups, J. High Energy Phys. 02 (2018) 163.

[27] O. Aharony, S. Giombi, G. Gur-Ari, J. Maldacena, and R. Yacoby, The thermal free energy in large $N$ ChernSimons-matter theories, J. High Energy Phys. 03 (2013) 121.

[28] S. Giombi, S. Minwalla, S. Prakash, S. P. Trivedi, S. R. Wadia, and X. Yin, Chern-Simons theory with vector Fermion matter, Eur. Phys. J. C 72, 2112 (2012).

[29] S. Jain, S. Minwalla, T. Sharma, T. Takimi, S. R. Wadia, and S. Yokoyama, Phases of large $N$ vector
Chern-Simons theories on $S^{2} \times S^{1}$, J. High Energy Phys. 09 (2013) 009.

[30] X. Arsiwalla, R. Boels, M. Marino, and A. Sinkovics, Phase transitions in q-deformed 2-D Yang-Mills theory and topological strings, Phys. Rev. D 73, 026005 (2006).

[31] M. R. Douglas and V. A. Kazakov, Large N phase transition in continuum QCD in two-dimensions, Phys. Lett. B 319, 219 (1993).

[32] R. Gopakumar and C. Vafa, M theory and topological strings. 1, arXiv:hep-th/9809187.

[33] V. Periwal, Topological Closed String Interpretation of Chern-Simons Theory, Phys. Rev. Lett. 71, 1295 (1993).

[34] S. Dutta and R. Gopakumar, Free Fermions and thermal AdS/CFT, J. High Energy Phys. 03 (2008) 011.

[35] P. Dutta and S. Dutta, Phase space distribution for two-gap solution in unitary matrix model, J. High Energy Phys. 04 (2016) 104.

[36] D. J. Gross and A. Matytsin, Some properties of large $N$ two-dimensional Yang-Mills theory, Nucl. Phys. B437, 541 (1995).

[37] M. Marino, Chern-Simons theory, matrix integrals, and perturbative three manifold invariants, Commun. Math. Phys. 253, 25 (2005).

[38] L. Rozansky, A contribution to the trivial connection to Jones polynomial and Witten's invariant of 3-d manifolds. 1, Commun. Math. Phys. 175, 275 (1996).

[39] T. Morita and K. Sugiyama, Multicut solutions in ChernSimons matrix models, Nucl. Phys. B929, 1 (2018).

[40] T. Morita and K. Sugiyama, Toward the construction of the general multicut solutions in chern-simons matrix models, J. High Energy Phys. 08 (2018) 168. 